\title{
Les coquillages marins de Praileaitz I (Deba, Gipuzkoa)
}

\author{
Praileaitz I (Deba, Gipuzkoa) itsas maskorrak \\ The marin shells from Praileaitz I (Deba, Gipuzkoa) \\ Las conchas marinas de Praileaitz I (Deba, Gipuzkoa)
}

MOTS-CLÉS : Objets de parure, Taphonomie, Littorinidae, Epipaéolithique.

GAKO-HITZAK: Apaingarriak, Tafonomia, Littorinidae, Epipaleolitoa.

KEYWORDS: Ornaments. Beads, Taphonomy, Littorinidae, Epipalaeolithic.

PALABRAS CLAVE: Objetos de adorno, Tafonomía, Littorinidae, Epipaleolítico.

\section{Solange RIGAUD ${ }^{(1-2)}$, Francesco D'ERRICO(3-4), Marian VANHAEREN ${ }^{(3)}$}

\section{RESUME :}

L'accumulation de coquilles à Praileaitz I, documente l'interaction des chasseurs-cueilleurs épipaléolithiques avec les environnements côtiers. La méthode développée pour étudier l'assemblage combine une analyse taxonomique à une analyse taphonomique, morphometrique et microscopique. Les coquilles appartiennent aux espèces $L$. saxatilis et $L$. obtusata. La comparaison avec les collections de référence modernes et archéologiques suggère que les coquilles ont été collectées au sein d'une thanatocénose, que l'accumulation est composée des plus petites coquilles disponibles au sein de l'environnement et que beaucoup d'entre elles présentent une cassure résultant de la prédation par les crabes. L'absence d'aménagement sur les coquilles et l'utilisation fréquente de ces gastéropodes pour produire des objets de parure, suggèrent que les coquilles ont été triées et rejetées sur le site, car impropres à la fabrication d'ornements corporels. L'absence d'autre type d'artefact associé aux coquillages souligne la nature hautement spécialisée de l'activité menée sur le site au cours de l'Epipaléolithique.

\section{LABURPENA:}

Praileaitz l-eko maskor metaketak, ehiztari-biltzaile epipaleolitikoek itsasbazterreko ingurumenarekin zuten interakzioa dokumentatzen du. Bilduma aztertzeko garatutako metodoak konbinatzen du analisi taxonomikoa, tafonomikoarekin, morfometrikoarekin eta mikroskopikoarekin. Maskorrak L. saxatilis-a eta L. obtusata espezieetakoak dira. Gaurko erreferentzia-bildumekin eta arkeologikoekin egindako erkaketak iradokitzen du maskorrak tanatozenosi baten barruan jaso zirela, metaketa osatuta dagoela ingurumenean dauden maskor txikienez, eta haietako askok haustura bat dutela karramarroen harraparitzagatik. Maskor horietan eraldaketa arrasto ezak, batetik, eta, bestetik, kontuan izanda barraskilo hauen ohiko erabilera apaindura-elementuak egiteko, iradokitzen dute, maskorrak aukeratu zirela eta, soineko apaingaiak lantzeko egokiak ez zirelako, lekuan bertan baztertuak izan zirela. Itsaskiekin lotutako beste mota bateko artefaktuak ez egoteak, Epipaleolito Aroan zehar, lekuan burututako jardueraren izaera oso espezializatua azpimarratzen du.

\section{ABSTRACT:}

The frequency of mollusks, shellfish, fish, seabirds, and marine mammal remains from archaeological sites in the Vasco-Cantabrian region of Northern Spain attests to the relatively intense exploitation of marine resources during the Upper Palaeolithic, Epipalaeolithic, and Mesolithic. Coastal resources were exploited for both utilitarian and symbolic purposes. Two gastropods (Littorina saxatilis/complexa/ arcana and Littorina obtusata), in particular, were almost continuously used for personal ornamentation. The shell accumulation at Praileaitz I, dated to c. 10,000 and 11,500 cal BP, provides new data concerning the interplay between Epipalaeolithic prehistoric hunter-gatherers and coastal environments. We combine analysis of the shell taxonomy with taphonomic, morphometric, and microscopic study of the shells assemblage. The shells almost exclusively belong to the species $L$. saxatilis/complexa/arcana and $L$. obtusata. They display no evidence of having been strung as ornaments. Comparison with modern and archaeological reference collections suggests that the shells were collected from a thanatocenosis, that the accumuation is composed of the smallest shells available at the time, and that many of them display a breakage resulting from crab predation. Given the frequent use of these shell species in the Vasco-Cantabrian region to produce personal ornaments, we propose that the assemblage represents an accumulation of shells discarded at the site because considered unsuitable for the manufacture of personal ornaments. This implies that the production of shell beads took place at different locations. The absence

\footnotetext{
(1) CNRS-UMI 3199 CIRHUS, 4 Washington Square North, New York, NY 10003, USA. srigaud@nyu.edu

(2) Department of Anthropology, New York University, New York, NY 10003, USA

(3) Univ. Bordeaux, CNRS-UMR 5199 PACEA, Équipe Préhistoire, Paléoenvironement, Patrimoine, avenue des Facultés, F-33405 Talence, France

(4) Evolutionary Studies Institute and DST/NRF Centre of Excellence in Palaeosciences, and School of Geosciences, University of the Witwatersrand, Private Bag 3, Wits, 2050, Johannesburg, South Africa.
} 
of other artefact types at the spot where the shells were found emphasises the highly specialized nature of bead production during the Epipalaeolithic.

\section{RESUMEN:}

La acumulación de conchas de Praileaitz I, documenta la interacción de cazadores-recolectores epipaleolíticos con el medio ambiente costero. El método desarrollado para estudiar el conjunto combina un análisis taxonómico con un análisis tafonómico, morfométrico y microscópico. Las conchas pertenecen a las especies $L$. saxatilis y L. obtusata. La comparación con las colecciones de referencia actuales y arqueológicas, sugiere que las conchas se recogieron en el seno de una tanatocenosis, que la acumulación está compuesta por las conchas más pequeñas existentes en el medio ambiente y que muchas de ellas tienen una rotura debido a la depredación por cangrejos. La falta de modificaciones de las conchas y el uso frecuente de estos caracoles para producir elementos de adorno, sugieren que las conchas fueron seleccionadas y desechadas en el sitio, por no ser aptas para la fabricación de adornos corporales. La ausencia de otro tipo de artefacto asociado a los mariscos subraya la naturaleza altamente especializada de la actividad llevada a cabo en el sitio durante el Epipaleolítico.

\section{1.- INTRODUCTION}

Les groupes humains ayant occupé la région Vascocantabrique durant le Paléolithique supérieur et le Mésolithique ont entretenu des relations étroites avec le milieu côtier, attestées à travers les traces récurrentes de l'exploitation des ressources marines, tant pour des fins économiques que symboliques (par ex. STRAUS et al., 2002, STRAUS, 1991, 2005, CORCHON et al., 2008, ALTUNAETXABE, MARIEZKURRENA-GAZTEARENA, 2010, ÁLVAREZ-FERNÁNDEZ, 2006, 2009, 2011a, GUTIÉRREZ-ZUGASTI, 2009a,b, 2011). Ces groupes ont recherché dans des ressources marines les apports nutritionnels nécessaires à leur subsistance, à travers la consommation de mollusques, de crustacés, de poissons et d'oiseaux de mer (ÁLVAREZ-FERNANDEZ, 2011a pour une synthèse), mais également les supports osseux nécessaires à la confection d'outillage, ou encore les matériaux voués à la confection d'ornements corporels tels que les coquillages et les dents de mammifères marins (CORCHON et al., 2008, ÁLVAREZ-FERNANDEZ, 2006, RIGAUD, GUTIÉRREZ-ZUGASTI, 2016). Les fouilles menées dans la cavité de Praileaitz I (PEÑALVER IRIBARREN, MUJIKA ALUSTIZA, 2003, PEÑALVER IRIBARREN, SAN JOSE SANTAMARTA, 2008) ont livré des occupations s'étalant des phases récentes du Paléolithique supérieur au Mésolithique. C'est au cours de ces fouilles qu'une accumulation de plusieurs centaines de coquillages marins a été identifiée au sein de la cavité (RIGAUD et al., 2014). La découverte de ces vestiges révèle une nouvelle facette de la relation homme-milieu côtier au cours de l'Épipaléolithique. L'analyse de cette accumulation, particulièrement bien préservée, offre une excellente opportunité pour mieux comprendre les liens complexes que ces populations ont tissés avec le milieu marin.

\subsection{Objectif de l'étude}

Notre analyse a comme objectif d'évaluer la cohérence temporelle de l'accumulation par sa mise en contexte stratigraphique et spatiale, de vérifier l'origine anthropique de l'accumulation de coquillages et de documenter les modifications éventuelles engendrées par cette dernière. Ces données, combinées à la datation directe du matériel, visent à proposer une attribution chrono-cultu- relle des vestiges et d'en comprendre la fonction. Les implications de nos résultats pour l'interprétation du statut du gisement au cours de l'occupation épipaléolithique et, plus généralement, sur la structuration des sociétés de cette période, font égalment parti de nos objectifs.

\subsection{Contexte archéologique}

Les concentrations de coquillages ont été observées lors de la fouille de la zone située entre la sortie du vestibule et l'entrée de la première salle intérieure de la cavité. La majorité des pièces a été récoltée au cours du tamisage des sédiments des fouilles des années 2003 et 2004. Les coquillages ont été retrouvés au sein d'une couche archéologique surmontée d'un niveau charbonneux attribué à l'Epipaléolithique et au dessus d'un niveau, attribué au Magdalénien inférieur, riche en pendentifs de pierre (PEÑALVER IRRIBARREN, MUJIKA ALUSTIZA, 2003, D'ERRICO, VANHAEREN, QUEFFELEC, 2017).

\section{2.- MÉTHODES}

\subsection{Identification taxonomique}

L'identification taxonomique a pris en compte la forme générale du test des coquilles et la caractérisation de leur ornementation permettant l'identification des coquillages au niveau du genre ou de l'espèce (e. g. POPPE, GOTO, 1993; HARASEWYCH, MORETZSOHN, 2010).

La nomenclature utilisée suit les classifications disponibles dans la base de données CLEMAM pour les coquillages marins [Check List of European Marine Mollusca http://www.somali.asso.fr/clemam/index.php?lang_=en].

Chaque espèce de coquillage se développant dans un environnement qui lui est propre, l'identification spécifique offre également des informations sur le biotope des spécimens exhumés. Dans le cas d'une accumulation anthropique et d'une acquisition directe des coquillages, cette information permet également d'identifier les environnements exploités par le groupe ayant occupé la cavité. Si les matériaux ont une origine allochtone et ont été indirectement acquis, il est aussi possible de caractériser la, ou les régions, avec lesquelles les populations en 
questions entretenaient des échanges (TABORIN, 1996, ÁLVAREZ-FERNÁNDEZ, 2001, VANHAEREN, D'ERRICO, 2005, FULLOLA et al., 2007).

\subsection{Analyse morphologique et morphométrique}

L'analyse métrique et la prise en compte de variables morphologiques contribuent à déterminer si les pièces accumulées dans le gisement sont représentatives d'une biocénose (communauté vivante) ou d'une thanatocénose (communauté morte) de la même espèce. Elles contribuent également à établir si les différences observées entre populations naturelles et échantillons archéologiques doivent être attribuées à une activité anthropique. Les variables enregistrées sont comparées à celles de référentiels de comparaison dont on connaît l'agent d'accumulation.

Quatre mesures ont été relevées à l'aide d'un pied à coulisse digital sur les coquillages de Praileaitz I : la longueur et largeur du coquillage, la largeur de l'avant dernière spire et la largeur de l'aperture (Fig. 1).

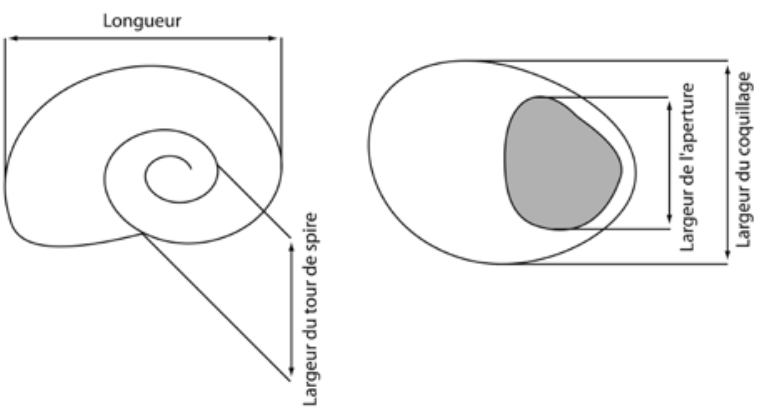

Fig. 1. Mesures enregistrées sur les gastéropodes de Praileaitz I. / Measurements recorded on the gastropods from Praileaitz I.

\subsection{Référentiels de comparaison}

Les référentiels de comparaison utilisés dans cette étude concernent deux espèces : Littorina obtusata et Littorina saxatilis.

Les deux référentiels actuels de $L$. obtusata ont été collectés sur la côte atlantique (Tabl. 1, VANHAEREN, D'ERRICO, 2003, VANHAEREN, 2002, 2010) au cours d'un ramassage exhaustif à vue au sein de thanatocénoses accumulées sur les plages (VANHAEREN, 2002).

Les Littorina obtusata de Praileaitz I ont également été comparées à 47 coquillages perforés de la même espèce provenant du niveau F (GrN 5817 : 17050 140 BP, UTRILLA, 1996) du gisement de Urtiaga (Pays basque, Espagne), ayant livré des indices d'occupations attribuées au Solutréen final et au Magdalénien inférieur. Les pièces de Prailiaitz I ont aussi été comparées à 29 coquillages de la même espèce, également perforés, provenant du niveau $\mathrm{E}$ du même gisement, attribué au

\begin{tabular}{|c|c|c|}
\hline & \multicolumn{2}{|l|}{\begin{tabular}{|l|}
$\begin{array}{l}\text { Plages } \\
\text { Localités }\end{array}$ \\
\end{tabular}} \\
\hline & Bonhomme & Souzeaux \\
\hline Espèces & Noirmoutier, France & Noirmoutier, France \\
\hline \multicolumn{3}{|l|}{ L. obtusata s. I. } \\
\hline citrina & 123 & 1 \\
\hline fusca & 72 & 60 \\
\hline olivacea & 2 & - \\
\hline aurantia et rubens & - & 8 \\
\hline reticulata & 14 & 1 \\
\hline alba & 4 & - \\
\hline alternata & - & 2 \\
\hline "hybrides" & 21 & 2 \\
\hline
\end{tabular}

Tabl. 1. référentiels de comparaison naturels actuels utilisés pour l'analyse des L. obtusata de Praileaitz I. / Modern reference collections of $L$. obtusata analysed in this study.

Magdalénien supérieur (UTRILLA 1996). Ce matériel est conservé au dépôt de fouille de Gordailua, Centre de Patrimoine Culturel Mobilier de Gipuzkoa à Irun.

Le référentiel naturel des Littotina saxatilis, composé de 366 coquillages, a été constitué par une collecte manuelle à vue de coquillages vivants, dans leur biotope, sur les falaises de la plage atlantique de Paloma (Tapia de Casariego, Asturies, Espagne). Les pièces ont été collectées en 20 minutes par deux personnes.

Deux Littorina saxatilis provenant des déblais du gisement de Urtiaga $(n=1)$, ayant livré des occupations magdaléniennes et aziliennes, et provenant du niveau $\mathrm{V}$ $(n=1)$ du gisement de Erralla (Pays basque, Espagne), ont également été analysées à titre comparatif. Le niveau $\mathrm{V}$ du gisement de Erralla est attribué au Magdalénien inférieur cantabrique (I-12868 : 16270 $\pm 240 \mathrm{BP}$; I-12551 :

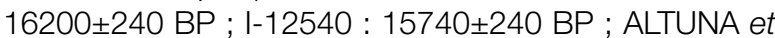
al., 1985, CAZALS, 2005, LANGLAIS, 2010). Les pièces sont conservées au dépôt de Gordailua à Irun.

\subsection{Préparation du matériel archéologique}

Les coquilles de Praileaitz I étaient couvertes d'un encroûtement calcitique très envahissant et plus ou moins épais, gênant l'identification spécifique, la mesure et l'analyse microscopique. Afin d'enlever le dépôt calcitique, les coquilles ont été plongées 1 à 2 minutes dans une solution composée pour $10 \%$ d'acide formique et 90\% d'eau. Lorsque des encroûtements étaient encore présents après ce traitement, les coquillages ont été laissés pendant une minute dans un bac à ultrasons. Des essais conduits au préalable sur quelques spécimens ont montré que les méthodes de nettoyage que nous avons appliquées ne produisent, même à l'échelle microscopique, aucun dommage aux coquillages. 


\subsection{Analyse microscopique}

La surface des coquillages porte des traces microscopiques témoignant des évènements qui se sont produits au cours de la vie du mollusque ou après sa mort. Dans le cas de coquillages récoltés et/ou modifiés par des préhistoriques, l'analyse microscopique peut nous informer sur les modalités de sélection et les lieux de collecte. Le mode d'utilisation des coquillages après leur ramassage et les modifications naturelles survenues à la suite de la perte ou de l'abandon des objets peuvent également être identifiés au cours de cette analyse (D'ERRICO et al., 1993, TABORIN, 1993, DUPONT, 2006).

Un microscope Leica Z6 APO équipé d'une caméra digitale a été utilisé pour documenter les modifications présentes sur la surface des coquillages de Praileaitz I.

Nous avons relevé la présence, la localisation et le degré de développement des modifications naturelles pouvant gêner la lecture des pièces (encroutements calcitiques, cassures). La présence des reliefs naturels des coquilles et leur degré de préservation ont également été enregistrés.

Les modifications d'origine naturelle ou anthropique telles que des fractures, des usures, des aménagements pour la suspension (perforation, sillon, résidu), la présence de pigment, et les traces d'utilisation ont également été systématiquement recherchées.

\subsection{Datation}

Une Littorina obtusata et une Littorina saxatilis provenant du centre de l'accumulation principale des coquillages ont été envoyées pour datation au Leibniz Labor für Altersbestimmung und Isotopenforschung Christian-Albrechts-Universität, Kiel. Ces coquillages n'ont été soumis, au préalable, à aucun traitement chimique.

\section{3.- RÉSULTATS}

\subsection{Provenance stratigraphique}

Les coquillages ont été découverts dans un niveau de sédiment marron situé au-dessous d'une large lentille de sédiment riche en charbon associé à des signatures de rubéfaction et attribuée à l'Epipaléolithique. Les coquilles proviennent de toute l'étendue de la couche marron et sont absentes de la couche sous-jacente, contenant des vestiges du Magdalénien inférieur. Dans le passage entre le vestibule et la première salle, les coquillages ont été récoltés de la limite inférieure de l'unité stratigraphique 5bis, qui marque la fin de la couche charbonneuse, jusqu'à la base de l'unité stratigraphique 9 . Dans la première salle intérieure, les coquillages proviennent des unités stratigraphiques 2 à 6 .

\subsection{Analyse spatiale}

Bien que la majorité des spécimens ait été récoltée au cours du tamisage des sédiments, les fouilles des années 2003 et 2004 ont permis l'enregistrement de la position spatiale exacte de $47 \%$ des pièces, ce qui permet une analyse de leur distribution (Fig. 2). Les coquillages proviennent d'une zone de $25 \mathrm{~m}^{2}$ située à l'entrée de la première salle. Les plus fortes concentrations s'observent dans les bandes C', D' et E', en particulier dans les carrés 10E', 12D' et dans les trois carrés proches 14E', 12E' et 8C' (Fig. 2).

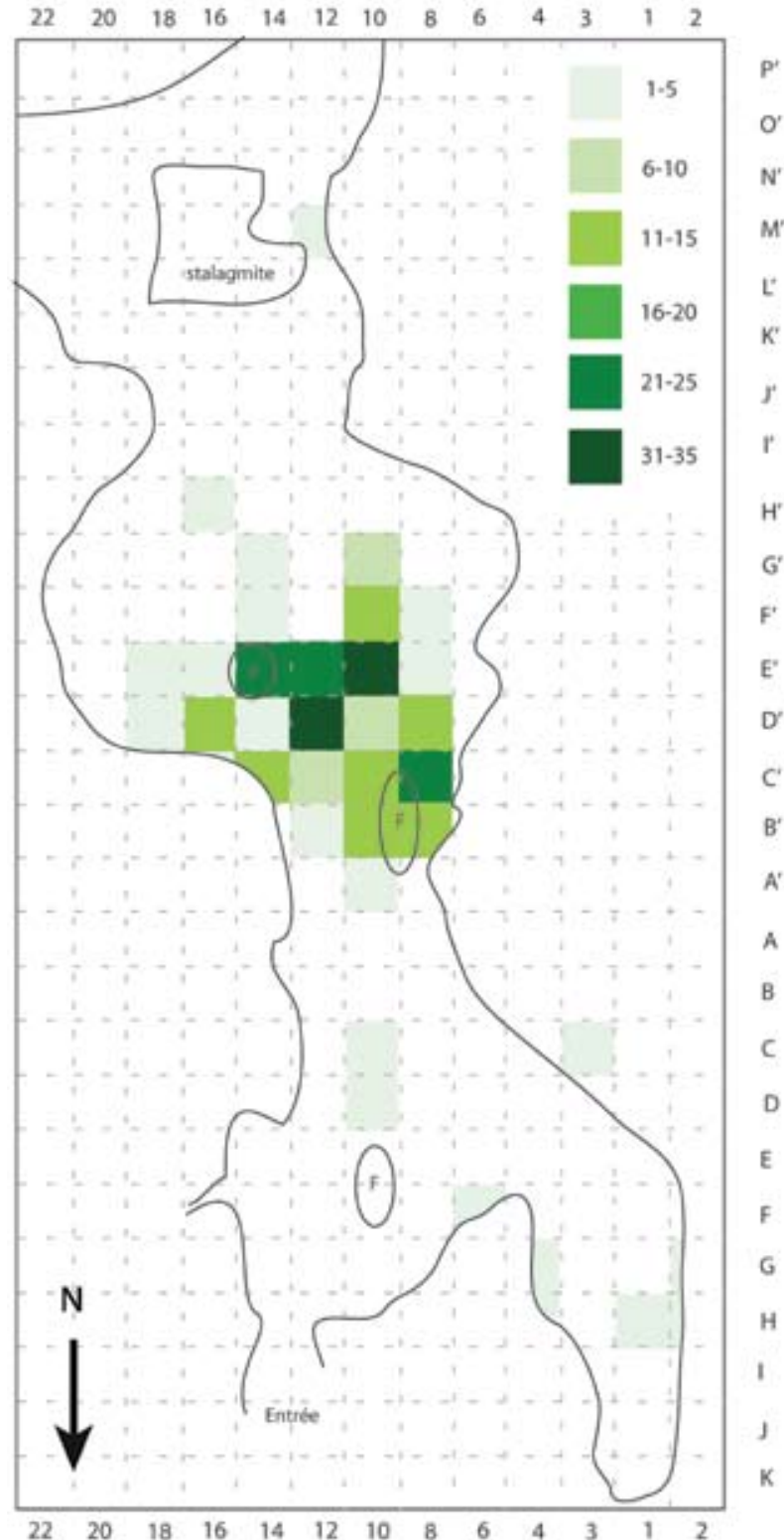

Fig. 2. Fréquence par mètre carré des coquillages marins au sein de la couche marron (modifié d'après RIGAUD et al., 2014). / Shell frequency/m2 in the brown layer (modified after RIGAUD et al., 2014). 
La projection verticale des pièces cotées dans les axes $x$ et $y$ révèle une dispersion sur $1 \mathrm{~m}$ de profondeur, entre $-250 \mathrm{~cm}$ et $-350 \mathrm{~cm}$. Au sein de ce volume, les coquillages sont concentrés dans une lentille de 30 à $50 \mathrm{~cm}$ d'épaisseur suivant un pendage orienté vers le sud-est (Fig. 3). Aucune différence significative ne s'observe entre les deux espèces dans leur distribution verticale.

\subsection{Les coquillages : identification, origines et description}

Un total de 237 coquilles a été collecté au cours de la fouille. Trois gastéropodes différents ont été identifiés : Littorina obtusata (Fig. 4), Littorina saxatilis (Fig. 5) et une Patella sp. qui n’a pu être spécifiquement déterminée.

\section{Z}
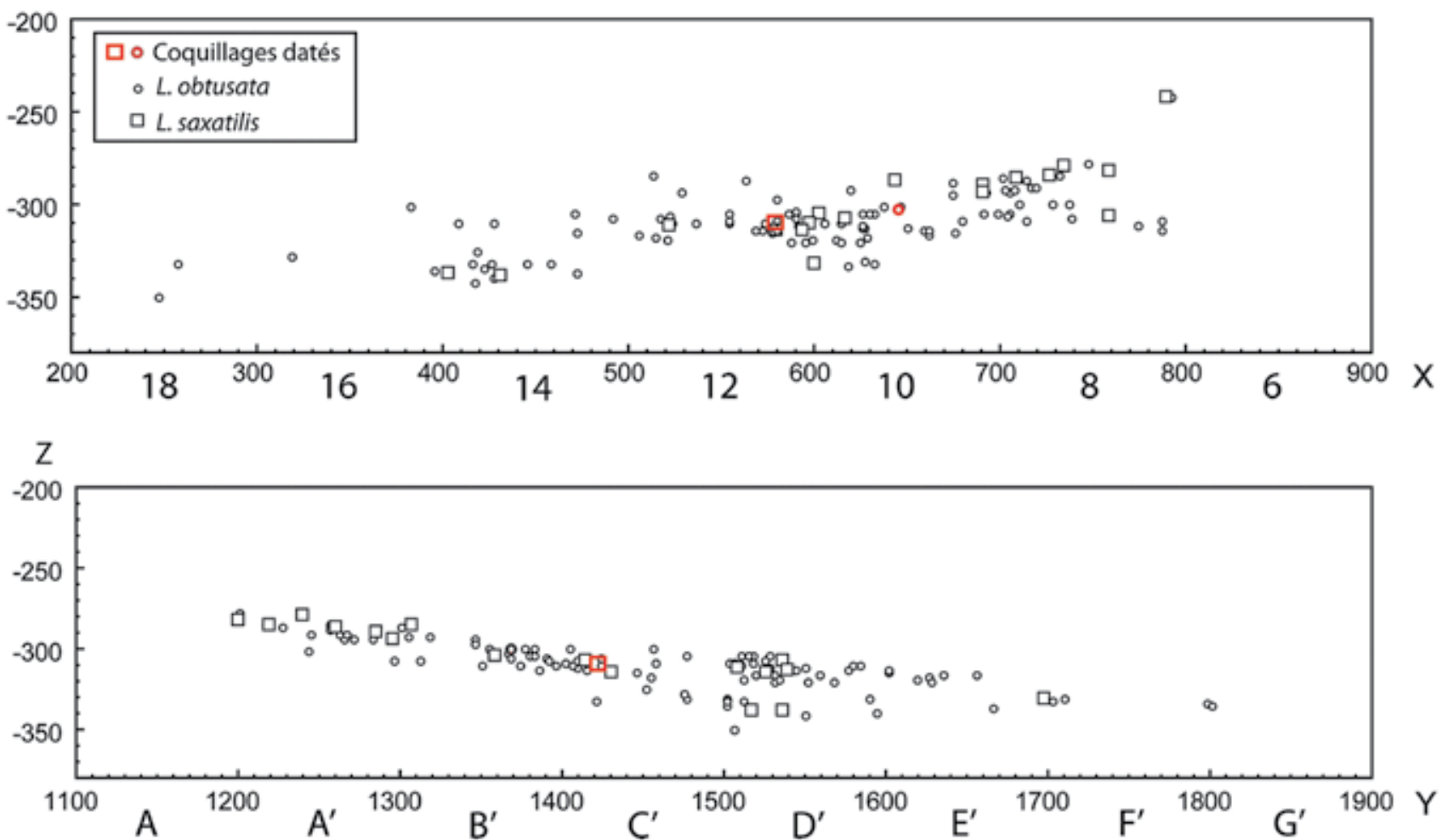

Fig. 3. Projections en $z$ et $x$ (en haut) et en z et y (en bas) des coquillages marins cotés pendant la fouille (modifié d'après RIGAUD et al., 2014). / Vertical distribution of the shells along the $x$ and y axes (modified after RIGAUD et al., 2014).

Les $L$. obtusata sont les plus représentées dans le gisement, suivie des L. saxatilis. (Tabl. 2).

\begin{tabular}{|l|c|c|}
\hline Espèce & NMI & \%NMI \\
\hline Littorina obtusata & 193 & 81.44 \\
\hline Complexe des Littorina saxatilis & 42 & 17.72 \\
\hline Patella sp. & 1 & 0.42 \\
\hline Gastéropode indet. & 1 & 0.42 \\
\hline
\end{tabular}

Tabl. 2. Proportion de coquillages par espèces découvertes sur le gisement de Praileaitz I. / Malacospectrum identified at Praileaitz I.

\section{- Littorina obtusata}

Les Littorina obtusata sont des petits gastéropodes dont la taille varie de 10 à 15 millimètres, mais qui peu- vent quelques fois atteindre $18 \mathrm{~mm}$. La coquille spiralée est presque lisse, plutôt globuleuse, mais aplatie au niveau de l'ouverture qui est ovale. Le dernier tour de spire constitue la partie la plus importante de la coquille. L'apex est aplati et peu visible. La coloration varie du jaune vif au brun roux presque noir, en passant par toutes les nuances de l'orange et du vert.

Elle peut être confondue avec Littorina fabalis qui présente une morphologie similaire bien que généralement plus petite et plus aplatie à l'apex. Cependant la haute variabilité intra-spécifique des deux coquillages ne permet pas une séparation claire entre les deux espèces du point de vue de leur forme (MONTEIRO CARVALHO, 2014). Seule la morphologie des organes reproducteurs des spécimens mâles permet de distinguer les deux espèces, diagnose qui ne peut être appliquée au matériel archéologique. 


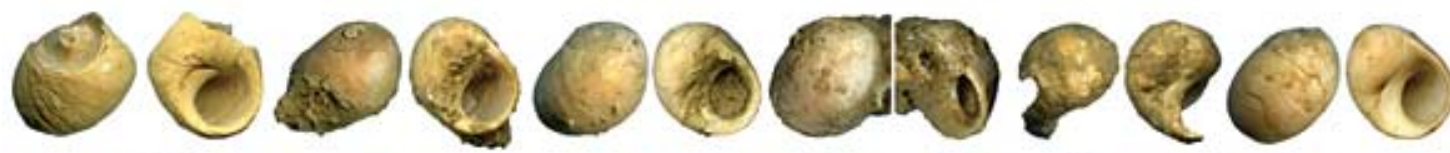

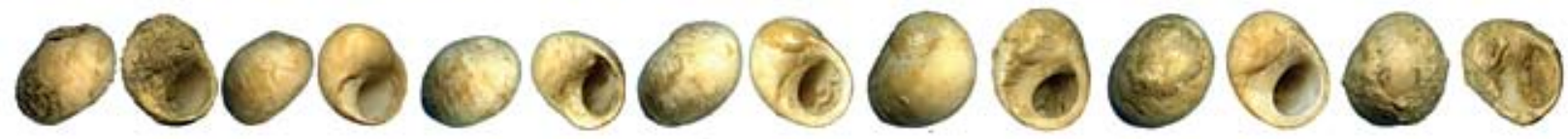
00000000000001 - 00 recocoropor 00000000000000 00000000076000Der $000000000000001 \mathrm{~cm}$ 0000000000001080 0000000000000000 0000000101000000 0000000000000000 07070091000000000000 0000000000000000 - 000000000000000

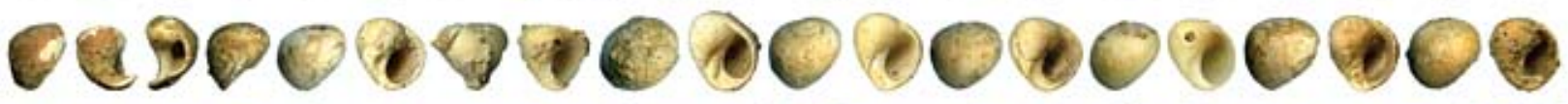
00000000000020000000

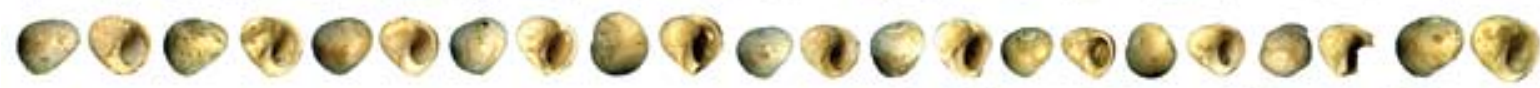

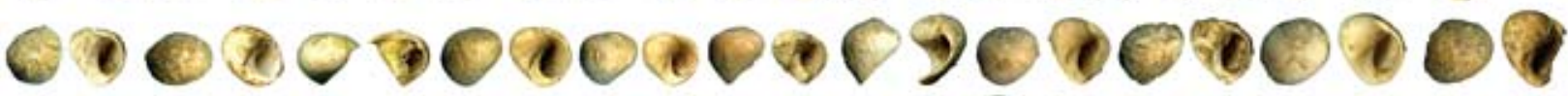

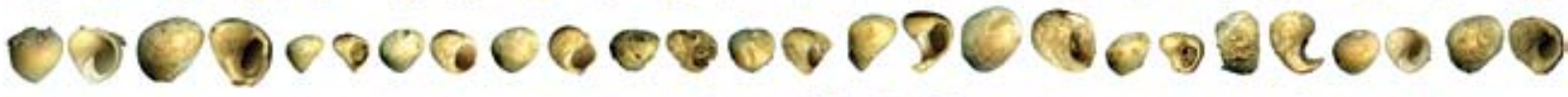
000007000000000000000000

Fig. 4. L. obtusata identifiées sur le gisement de Praileaitz I. / L. obtusata identified at Praileaitz I. 


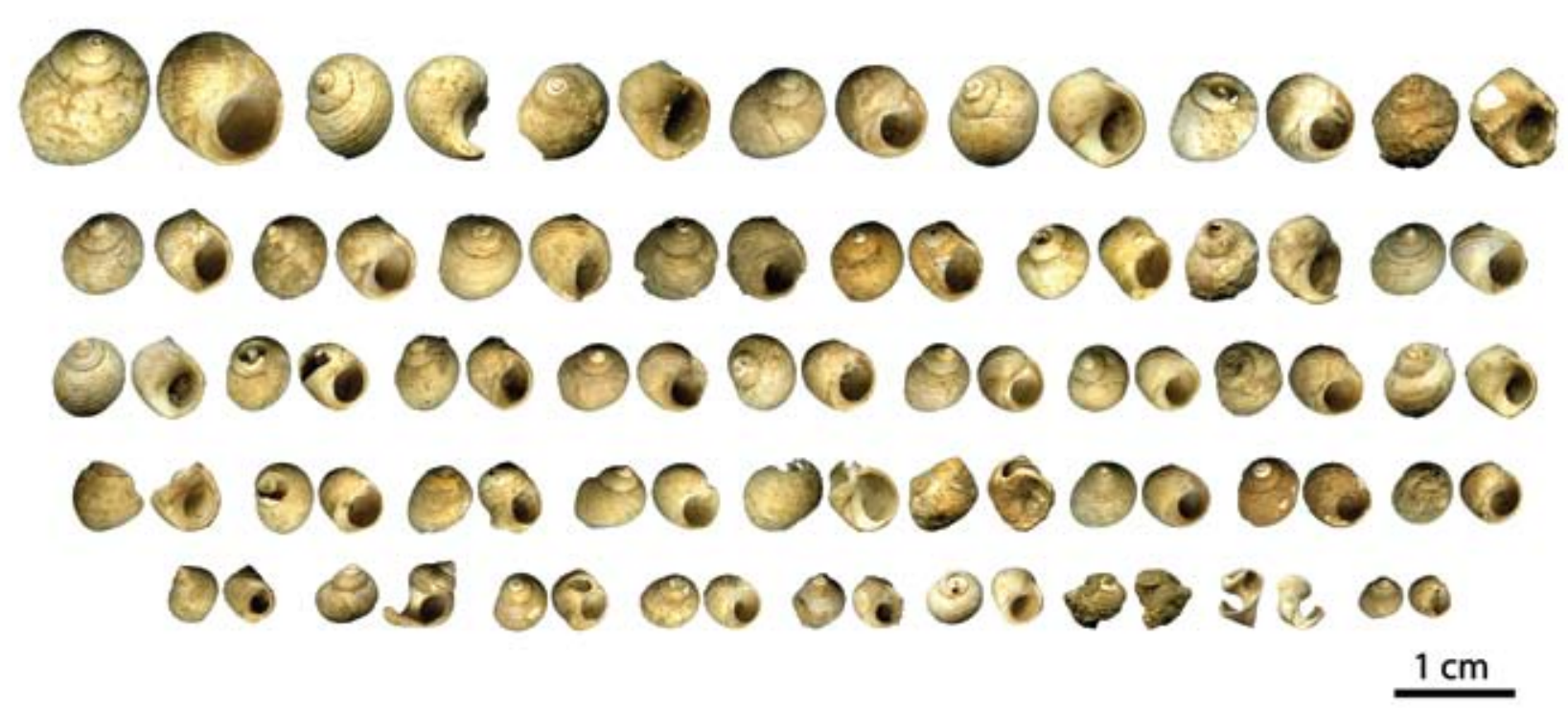

Fig. 5. L. saxatilis identifiées sur le gisement de Praileaitz I (modifié d'après RIGAUD et al., 2014). / L. saxatilis identified at Praileaitz I (modified after RIGAUD et al., 2014).

Les deux espèces sont actuellement présentes le long de la façade atlantique, du nord de la Norvège jusqu'au sud de l'Espagne. Elle sont également présentes en mer du Nord et en mer Baltique.

Littorina obtusata se développe sur les frondes des algues appartenant aux espèces Fucus vesiculosus et Ascophyllum nodosum qui couvrent des fonds rocheux. Cette espèce intertidale, essentiellement distribuée dans des zones de marnage abritées, peut se rencontrer dans des zones plus ou moins saumâtres tels que des estuaires (POPPE, GOTO, 1991). Littorina fabalis peut être trouvée dans des zones plus exposées que les L. obtusata (REID, 1996).

\section{- Le complexe des Littorina saxatilis}

Ce complexe regroupe les espèces $L$. compressa, $L$. arcana et $L$. saxatilis. Ces gastéropodes ont un apex pointu et comprennent trois à cinq tours de spires séparés par une suture profonde. Le test est couvert de côtes et de sillons réguliers. La coquille mesure en général de 8 à 14 millimètres mais peut exceptionnellement atteindre 18 millimètres. La couleur de fond est blanchâtre, jaune-orangé ou crème et jusqu'à gris-verdâtre à brun-noir (GAILLARD, 1977).

La morphologie extrêmement proche des coquilles de ces trois espèces n'a pas permis de les distinguer au sein des premières classifications établies sur la base de la morphologie des coquilles (REID, 1996, PANOVA et al., 2011). Ce sont les observations, plus tardives, des caractères reproductifs des femelles de chacune de ces espèces, qui ont permis de les identifier, mais ces critères ne sont pas applicables au matériel archéologique (CONDE-PADIN et al,. 2007, PANOVA et al., 2011). La coquille de $L$. compressa peut éventuellement se distinguer par la présence de sillons spiralés très fins (CONDEPADIN et al., 2007).

Les espèces de gastéropodes appartenant au complexe des Littorina saxatilis sont relativement ubiquistes. Elles sont présentes le long de la façade atlantique du nord de la Norvège jusqu'au Sud de l'Espagne, et sont également présentes dans la Manche et en Mer Baltique. Elles vivent dans une variété d'habitats tels que des falaises, des côtes rocheuses, et se rassemblent sous les cailloux et au sein de fissures et crevasses (PANOVA et al., 2011). Elles sont également présentes dans des zones plus ou moins vaseuses, de faible salinité telles que les lagons et les estuaires (PANOVA et al., 2011). Ces espèces s'observent depuis le haut du niveau des pelvéties (Pe/vetia canaliculata), dans la zone des embruns, jusqu'au Fucus spiralis, médiolittoral supérieur et supralittoral. Elles s'accommodent aux zones à faible et relativement fort hydrodynamisme.

\begin{tabular}{|c|c|c|c|c|c|}
\hline Lab code & échantillon & pMC corrigé & Âge conventionnel & $\delta \mathbf{1 3 C}(\%)$ & cal BP Corr \\
\hline KIA-39657 & L. saxatilis 0,8 mg C & $31.34 \pm 0.16$ & $9320 \pm 40 \mathrm{BP}$ & $-3.15 \pm 0.12$ & $10571-10252$ \\
\hline IA-39658 & L. obtusata 1,1 mg C & $26.86 \pm 0.16$ & $10560 \pm 50 \mathrm{BP}$ & $-0.50 \pm 22$ & $12621-12114$ \\
\hline
\end{tabular}

Tabl. 3. Âges radiocarbone obtenus à partir de deux coquillages marins de Praileaitz I. / Radiocarbon ages obtained on two marine shells found at Praileaitz I. 


\subsection{Datation}

Deux dates ${ }^{14} \mathrm{C}$ ont été obtenues par accélérateur sur une coquille de chacune des espèces de Littorines (Tabl. 3). Ces dates ont été calibrées et corrigées en considérant l'effet réservoir marin en utilisant la courbe de calibration Intcal 13 (programme de calibration Oxcal 4.2, REIMER et al., 2013) et un delta $R$ de $74 \pm 50$, calculée à partir de l'échantillon de Saint-Jean-de-Luz (Marine Reservoir Correction Database, TISNERAT-LABORDE, 2010).

\subsection{Analyse morphometrique}

\section{- Littorina obtusata}

La comparaison des L. obtusata de Praileaitz I à celles des référentiels montre que les coquillages accumu-

\begin{tabular}{|c|c|c|c|c|c|}
\hline Site & N & moyenne & StdDv & max & min \\
\hline Praileaitz I & 122 & 8,97 & 2,18 & 14,71 & 4,47 \\
\hline Urtiaga F & 43 & 15,53 & 2,22 & 19,80 & 8,75 \\
\hline Urtiaga E & 7 & 13,78 & 2,93 & 15,97 & 8,49 \\
\hline Bonhomme & 270 & 11,66 & 2,49 & 17,44 & 6,55 \\
\hline Souzeaux & 58 & 12,94 & 1,19 & 15,03 & 9,06 \\
\hline
\end{tabular}

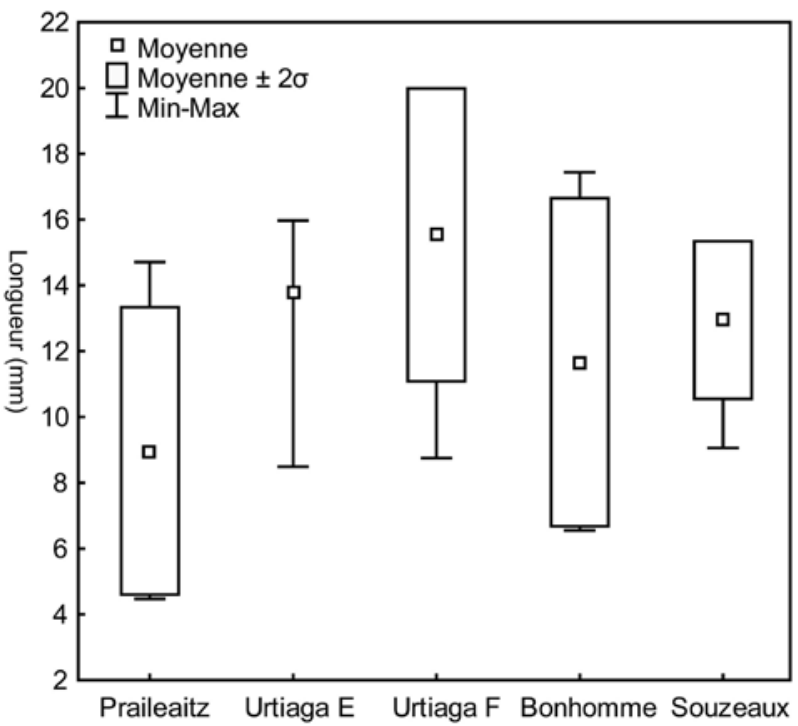

lés à Praileaitz I sont significativement plus petits que les coquillages constituant les référentiels de comparaison actuels et archéologiques (Man-Whitney U-test : $p<0.05)$. Les plus petites longueurs et largeurs du premier tour de spire des coquillages appartenant aux référentiels se superposent à la variabilité métrique des coquillages de Praileaitz I. En revanche, les coquillages aux plus petites dimensions présents à Praileaitz I sortent de la variabilité métrique identifiée au sein des référentiels de comparaison actuels et archéologiques (Fig. 6)

La mise en relation de ces variables montre les mêmes tendances : la distribution des coquillages de Praileaitz I ne couvre que la variabilité métrique des plus petits coquillages représentés dans les référentiels actuels de comparaison et des référentiels archéologiques de Urtiaga F et E (Fig. 7).

\begin{tabular}{|c|c|c|c|c|c|}
\hline Site & N & moyenne & StdDv & max & min \\
\hline Praileaitz I & 122 & 4,48 & 1,13 & 7,51 & 2,49 \\
\hline Urtiaga F & 43 & 7,22 & 1,13 & 9,08 & 4,36 \\
\hline Urtiaga E & 7 & 5,90 & 1,60 & 7,43 & 3,02 \\
\hline Bonhomme & 270 & 6,13 & 1,37 & 9,82 & 3,07 \\
\hline Souzeaux & 58 & 7,16 & 0,73 & 8,11 & 4,68 \\
\hline
\end{tabular}

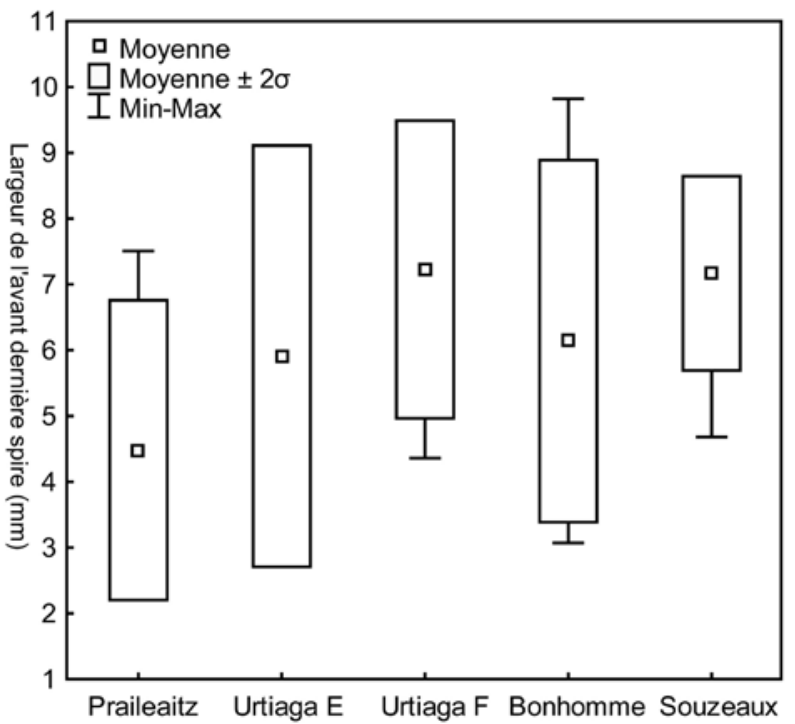

Fig. 6. Comparaison de la longueur du coquillage et de la largeur du premier tour de spire des $L$. obtusata de Praileaitz I avec les mesures enregistrées sur les référentiels de comparaison actuels (Bonhomme, Souzeaux, Noirmoutier) et archéologiques (Urtiaga E et F) (modifié d'après RIGAUD et al., 2014). / Comparison between shell length and first spiral whorl width of L. obtusata from Praileaitz I, modern (Bonhomme, Souzeaux, Noirmoutier), and archaeological reference collections (Urtiaga E and F) (modified after RIGAUD et al., 2014). tilis

- Les coquillages du complexe des Littorina saxa-

Les coquilles appartenant au complexe des L. saxatilis provenant du gisement de Praileaitz I partagent une grande partie de leur variabilité métrique avec le référentiel actuel de Paloma (Fig. 8, Fig. 9). Cependant, la com- paraison de la largeur des coquilles et de la largeur de leur aperture montre que les coquilles de Praileaitz I sont significativement plus grandes que les coquilles du référentiel actuel de Paloma (Man-Whitney U-test : $p<0.05$ ). Les coquilles aux plus petites dimensions du référentiel actuel de Paloma ne sont pas représentées dans la 

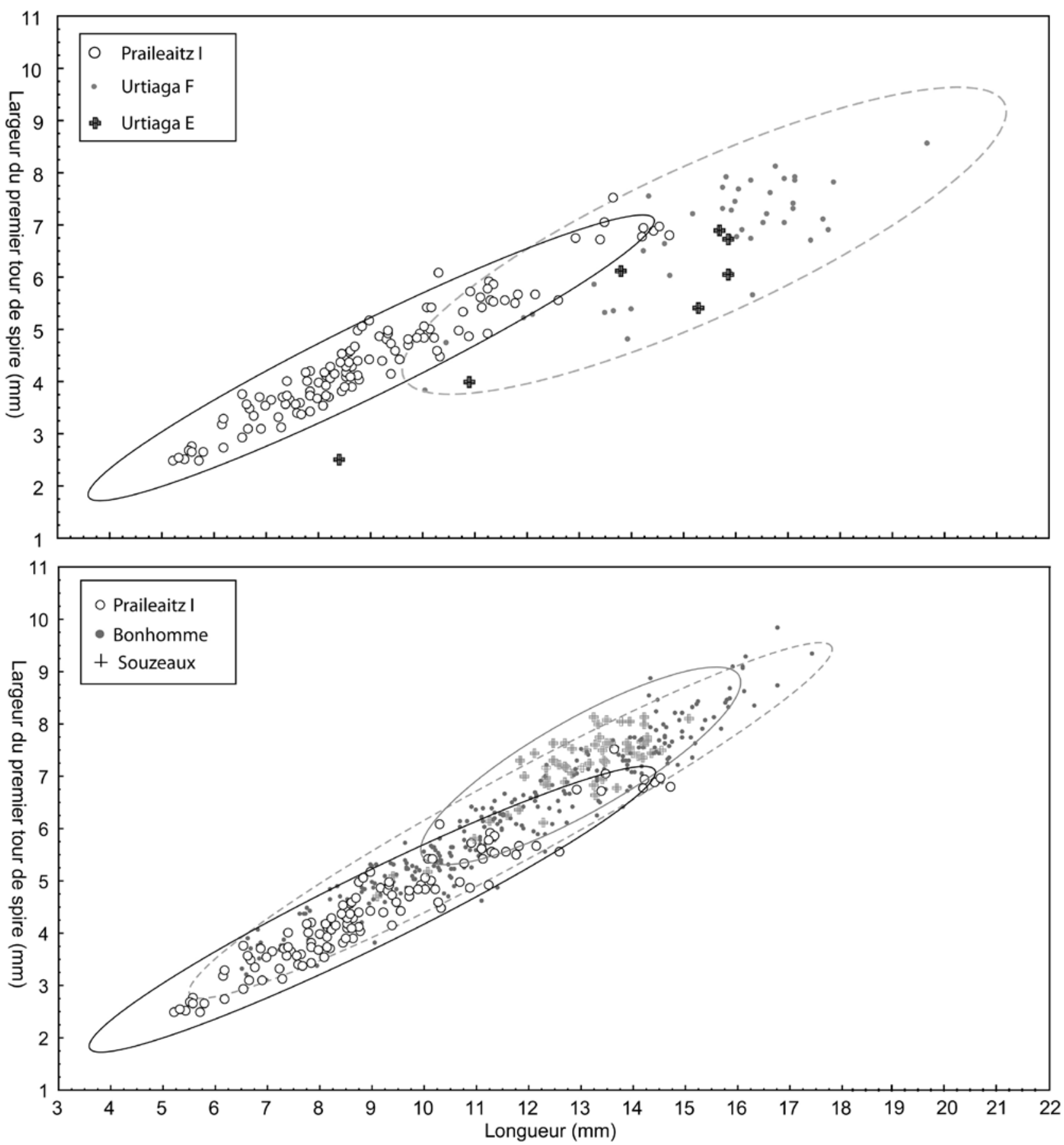

Fig. 7. Nuage de point mettant en relation la longueur et de la largeur du premier tour de spire des $L$. obtusata de Praileaitz I et des référentiels de comparaison actuels (Bonhomme, Souzeaux) et archéologiques (Urtiaga E et F) (modifié d'après RIGAUD et al., 2014). / Scatterplot of the length and first spiral whorl width recorded on the L. obtusata from Praileaitz I, modern (Bonhomme, Souzeaux, Noirmoutier), and archaeological reference collections (Urtiaga E and F) (modified after RIGAUD et al., 2014).

collection de Praileaitz I (Fig. 8, Fig. 9). Inversement, les coquilles les plus grandes de Praileaitz I sortent de la variabilité métrique enregistrée sur le référentiel actuel de Paloma (Fig. 8, Fig. 9).

\subsection{Analyse technologique}

L'analyse microscopique n'a permis de mettre en évidence aucun aménagement d'origine anthropique. En revanche, des cassures sur le bord avant de l'aperture 


\begin{tabular}{|c|c|c|c|c|c|}
\hline Site & N & Moyenne & StdDv & $\max$ & $\min$ \\
\hline PraileaitzI & 29 & 7,69 & 1,75 & 13,63 & 4,35 \\
Paloma & 364 & 6,40 & 1,16 & 9,79 & 2,52 \\
\hline
\end{tabular}

\begin{tabular}{|c|c|c|c|c|c|}
\hline Site & N & Moyenne & StdDv & $\max$ & $\min$ \\
\hline Praileaitzl & 29 & 5,41 & 1,36 & 9,98 & 3,13 \\
\hline Paloma & 364 & 4,70 & 0,82 & 6,82 & 1,38 \\
\hline
\end{tabular}
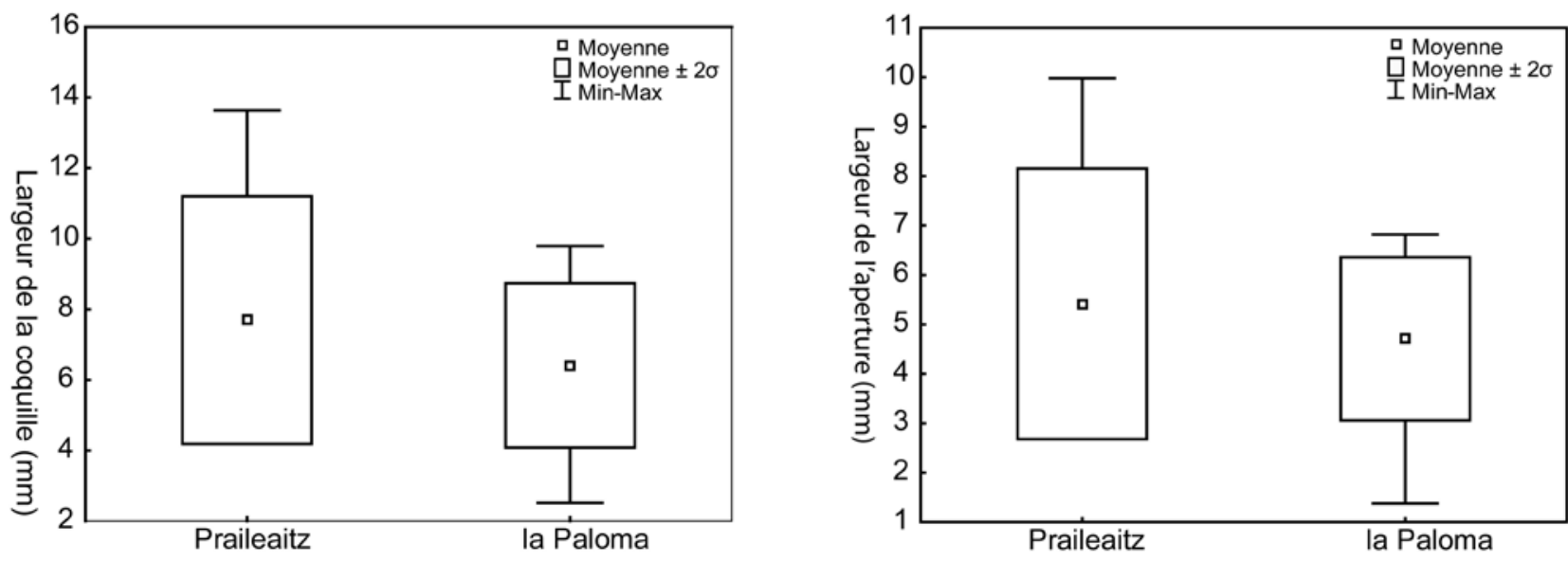

Fig. 8. Comparaison de la largeur des coquilles et de la largeur de l'aperture enregistrées sur les pièces attribuées au complexe de $L$. saxatilis de Praileaitz I et du référentiel actuel de Paloma (modifié d'après RIGAUD et al., 2014). / Comparison of the shell and aperture width of $L$. saxatilis complex from Praileaitz and the modern reference collection gathered at Paloma (modified after RIGAUD et al., 2014).

ont été observées sur $56 \%(n=138)$ des 235 coquilles des deux espèces de littorines. Ces cassures se déclinent en 4 types différents, envahissant plus ou moins la face dorsale du test (Fig. 10).

Le premier type de cassure est caractérisé par un esquillement peu envahissant du bord de l'aperture qui apparaît irrégulier (L. obtusata : $n=46$; L. saxatilis : $n=5$, Fig. $11 \mathrm{~d}$, e, f). Sur le second type, une encoche envahit la coquille, depuis le bord de l'aperture jusqu'à l'avant de la face dorsale du coquillage ( $L$. obtusata : $n=61$; L. saxatilis : n=9, Fig. 11 g, h, i). Le type 3 présente une encoche plus importante qui se développe légèrement sur la face dorsale de la coquille ( $L$. obtusata : $n=4$; L. saxatilis : $n=1$, Fig. $11 \mathrm{j}, \mathrm{k}, \mathrm{l}, \mathrm{m})$. Sur les coquillages présentant des cassures de type 4, l'encoche est plus importante et se découpe nettement sur la face dor-

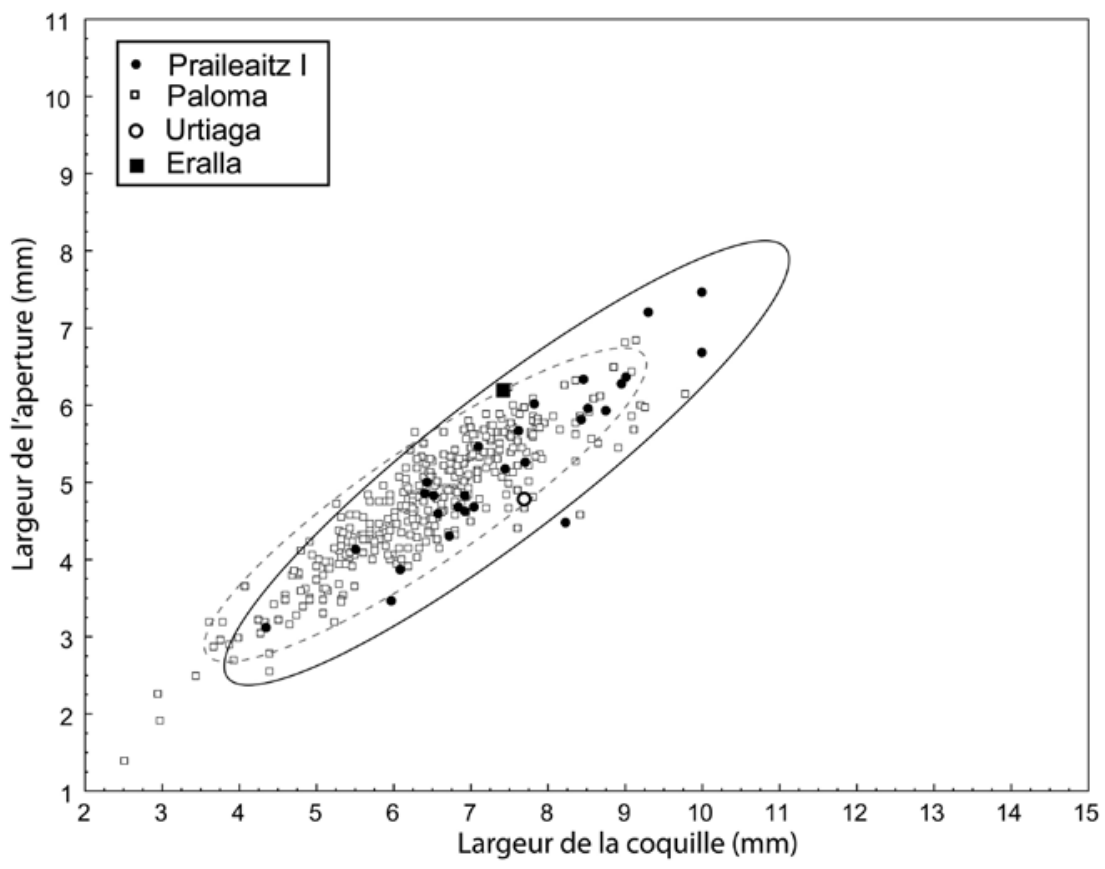

Fig. 9. Comparaison de la distribution de la largeur des coquilles et de la largeur de leur aperture enregistrées sur les pièces attribuées au complexe des $L$. saxatilis de Praileaitz I et du référentiel actuel de Paloma (modifié d'après RIGAUD et al., 2014). / Scatterplot of the shell and aperture width recorded on the $L$. saxatilis complex from Praileaitz I and the modern reference collection gathered at Paloma (modified after RIGAUD et al., 2014). 

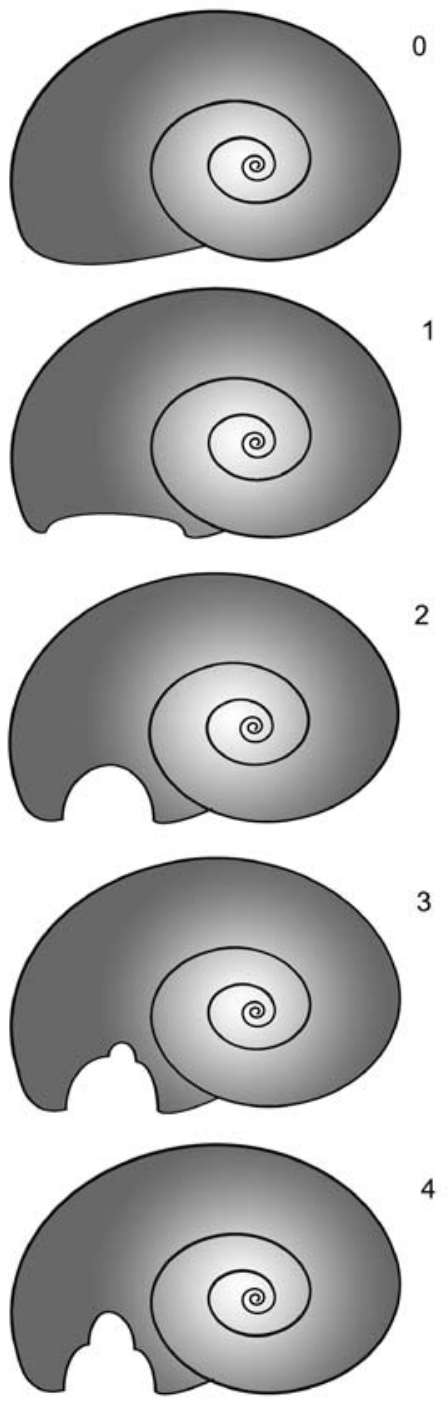

Fig. 10. Schéma des quatre types de cassures observés sur le bord de l'aperture des littorines de Praileaitz I. / Morphology of the four types of fractures observed on the Littorina sp. apertures from Praileaitz I.

sale de la coquille (L. obtusata : $n=2 ; L$. saxatilis : $n=2$, Fig. 110, p).

Deux pièces présentent une cassure sur l'avant de l'aperture qui se propage en biais et s'étend le long des spires (Fig. $11 \mathrm{~s}, \mathrm{t}$ ). Deux autres pièces montrent une petite perforation conique localisée sur la face ventrale de la coquille (Fig. 11 q, r). Ces perforations sont typiques des traces d'un prédateur ayant consommé le mollusque.

\section{4.- DISCUSSION}

Les résultats obtenus par nos différentes analyses permettent d'aborder les questions posées au début de cette étude, à savoir à quand remonte l'accumulation des littorines découvertes à Praileaitz I et quelles sont les raisons de sa présence dans cette cavité.

Ces deux espèces de coquillage sont largement documentées dans la région vasco-cantabrique en tant que support pour la réalisation d'objets de parure au sein des sociétés du Paléolithique supérieur, de l'Épipaléolithique et du Mésolithique. Des L. obtusata perforées ont été recensées au sein d'une cinquantaine d'occupations alors que des $L$. saxatilis perforées sont identifiées au sein de 7 occupations seulement (ÁLVAREZ-FERNANDEZ, 2006). Parmi ces gisements 9 sont attribués à l'Epipaléolithique (ÁLVAREZ-FERNANDEZ, 2006).

Par ailleurs, ces deux espèces non perforées sont régulièrement documentées au sein de gisements de la région vasco-cantabrique ayant livré des occupations attribuées à la fin du Paléolithique supérieur, à l'Epipaléolithique et au Mésolithique, et dont les vestiges attestent de l'exploitation des ressources marines à des fins alimentaires (ÁLVAREZ-FERNÁNDEZ, 2011, GUTIÉRREZ-ZUGASTI, 2011). Dans ces gisements les spectres malacologiques sont majoritairement dominés par les Patella sp. suivies des Littorina littorea (ÁLVAREZ-FERNÁNDEZ, 2009, 2010, 2011a ; GUTIÉRREZZUGASTI, 2009a, b, 2011). Les Littorina obtusata et les Littorina saxatilis dépassent très rarement une proportion supérieure à $0.8 \%$ du NMI (ÁLVAREZ-FERNÁNDEZ, 2009, 2010, 2011a, GUTIÉRREZ-ZUGASTI, 2009a, b, 2011). Seul le niveau $E$ du gisement de la Garma A, attribué au Gravettien, a livré un spectre malacologique contenant plusieurs dizaines de $L$. obtusata et $L$. fabalis représentant environ $20 \%$ du NMI. Cependant, sur ce gisement, les littorines appartenant à ces deux espèces sont très majoritairement aménagées en objet d'ornementation corporelle (ÁLVAREZ-FERNÁNDEZ, 2007, 2011a). En résumé, lorsque ces coquillages sont présents à l'état non transformé dans les gisements, ils représentent une part anecdotique du spectre malacologique des espèces consommées. Lorsque la proportion est plus grande, ils sont en général transformés en objet de parure. La présence quasi-exclusive de L. obtusata et de L. saxatilis à Praileaitz I (une seule Patella sp. a été identifiée dans le niveau) et l'absence d'aménagement de ceux-ci pour la suspension, ne rappellent aucune des accumulations de ces coquillages ayant fait l'objet d'étude jusqu'à présent (Fig. 12).

\subsection{Origine de l'accumulation}

Praileaitz I est actuellement localisé à une altitude de $50 \mathrm{~m}$ au dessus du niveau de la mer et à une distance d'environ $4.5 \mathrm{~km}$ de la côte. II y a 11000 ans le niveau marin était $60 \mathrm{~m}$ plus bas de l'actuel (LAMBECK, CHAPPEL, 2001, LAMBECK et al., 2002). En considérant la morphologie de la plateforme continentale dans cette zone de la côte (GALPARSORO et al., 2010, LEORRI et al., 2012), la mer devait se trouver à cette époque à environs $13 \mathrm{~km}$ de l'entrée de la grotte. 


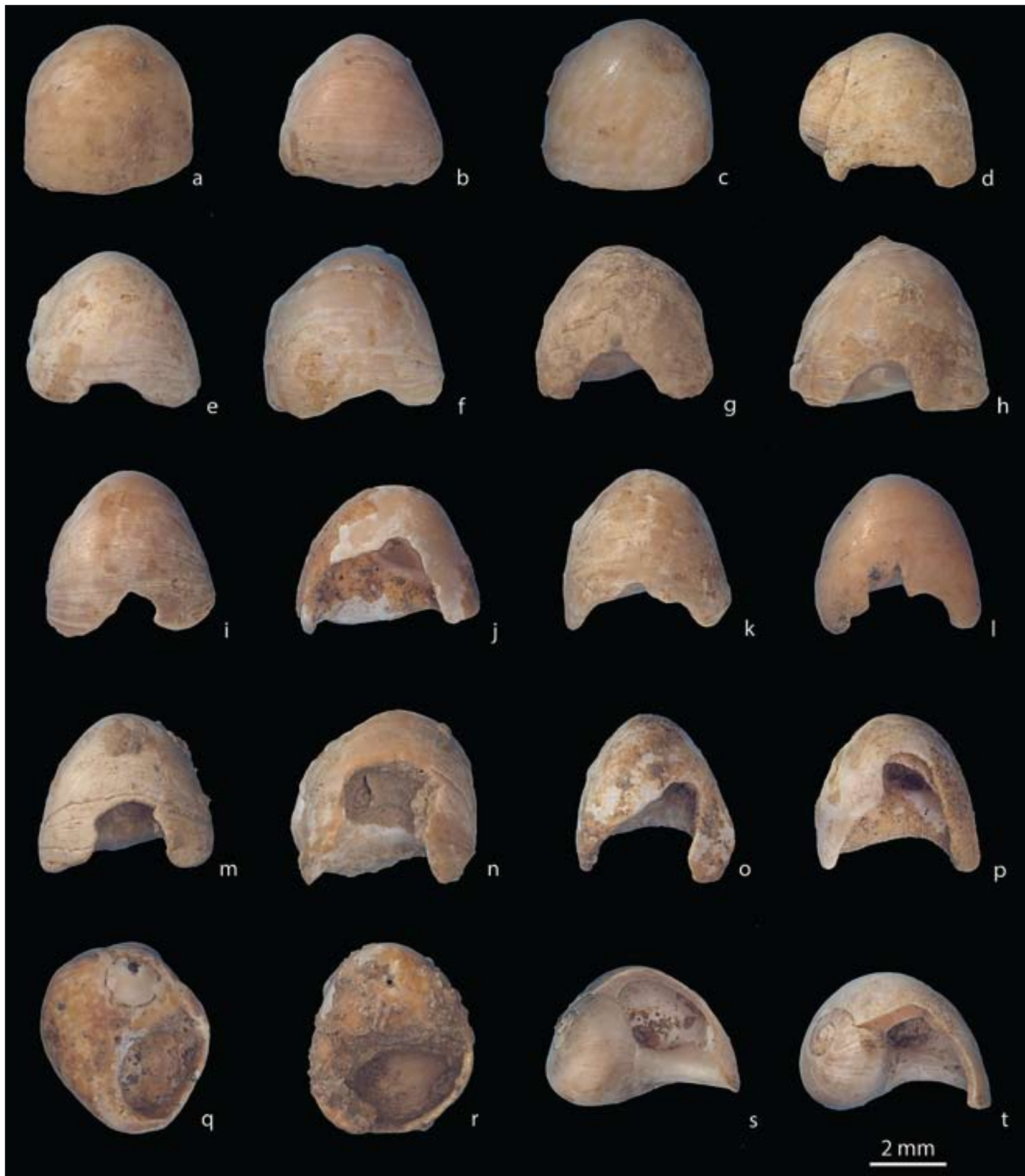

Fig. 11. $a, b$ : exemple d'apertures intactes ; c-p et $s, t$ : types de fractures identifiées sur les littorines de Praileaitz $l ; q, r$ : perforations dues à un prédateur (modifié d'après RIGAUD et al., 2014). / a, b: unbroken (a,b) and broken (c-p and s, t) shell apertures and perforation resulting from mollusk predation observed on the Littorina from Praileaitz I (modified after RIGAUD et al., 2014).

Les principaux prédateurs des espèces de Littorines représentées dans les environnements côtiers actuels sont des poissons intertidaux, des crabes et des petits échassiers tels que la bécasse maubèche (Calidris canutus) notamment, ou encore des huîtriers qui peuvent diversifier leur diète avec la consommation de ces petits gastéropodes selon le besoin (TREWIN, WELSH, 1976, JOHANNESSON, EKENDAHL, 2002, NILES et al., 2006). Les crabes et les poissons intertidaux ne peuvent avoir contribué à l'accumulation des coquilles dans 


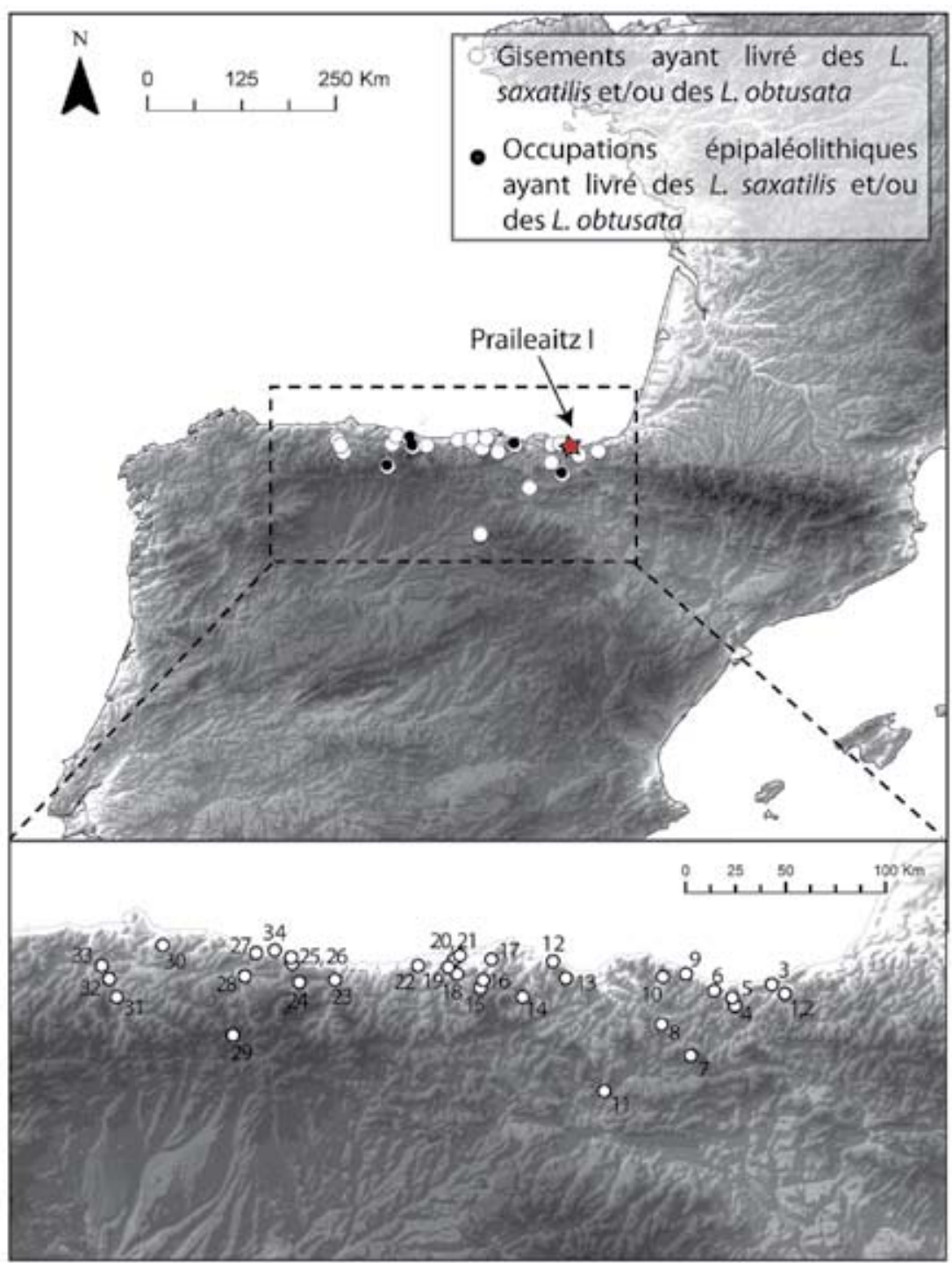

1) Aitzbitarte III, 2) Aitzbitarte IV, 3) Praileaitz I, 4) Erralla, 5) Amalda, 6) Urtiaga, 7) Anton Koba II, 8) Bolinkoba, 9) Lumentxa, 10) Santimanine, 11) Fuente Hoz 1, 12) El Cuco, 13) San Juan, 14) El Mirón, 15) El Piélago II, 16) El Rascaño V, 17) La Garma A, 18) Morín, 19) El Pendo 2, 20) El Juyo, 21) El Ruso, 22) Venta del Cuco, 23) La Paloma, 24) Los Canes, 25) La Poza I'Egua, 26) La Riera, 27) Tito Bustillo, 28) El Buxu, 29) Los Azules I, 30) El Conde, 31) Entrefoces, 32) Las Caldas, 33) La Ancenia, 34) El Mazo
Fig. 12. Distribution des gisements attribués au Paléolithique supérieur, à l'Azilien et au Mésolithique ayant livré des L. obtusata et des L. saxatilis perforées dans la région vasco-cantabrique (ÁLVAREZ-FERNÁNDEZ, 2006, GUTIÉRREZ-ZUGASTI et al., 2013, RIGAUD, GUTIÉRREZ-ZUGASTI, 2016). I Geographic distribution of the Vasco-Cantabrian sites with perforated L. obtusata and L. saxatilis attributed to the Upper Palaeolithic, Epipalaeolithic and Mesolithic (ÁLVAREZFERNÁNDEZ, 2006, GUTIÉRREZ-ZUGASTI et al., 2013, RIGAUD, GUTIÉRREZ-ZUGASTI, 2016). une cavité aussi éloignée du littoral. Les données éthologiques disponibles concernant les oiseaux mentionnés plus haut indiquent que ces espèces, principalement migratrices, nichent majoritairement sur la zone intertidale pendant leur période de migration en Europe. Au cours de la consommation du gastéropode, la coquille est cassée, le mollusque consommé et l'opercule régurgité (TREWIN, WELSH, 1976).

La localisation de la grotte hors de la zone intertidale, la nature non-fragmentaire des vestiges, leur localisation éloignée de l'entrée de la grotte et le caractère quasi mono-spécifique de l'accumulation sont incompatibles avec les caractéristiques éthologiques des prédateurs mentionnés ci-dessus. En somme, bien que des facteurs na- turels d'accumulation de ces coquillages sur le continent soient connus, aucun d'entre eux ne peut être raisonnablement évoqué pour expliquer une telle accumulation au sein de la cavité de Praileaitz I. La relative proximité du gisement de la ligne côtière suggère que les coquillages ont pu aisément être collectés et abandonnés à Praileaitz I par un groupe de chasseurs-cueilleurs ayant occupé la cavité.

\subsection{Acquisition}

Les coquillages ont pu être collectés vivants ou prélevés au sein d'une thanatocénose. Les données éthologiques disponibles pour les deux espèces de littorines 


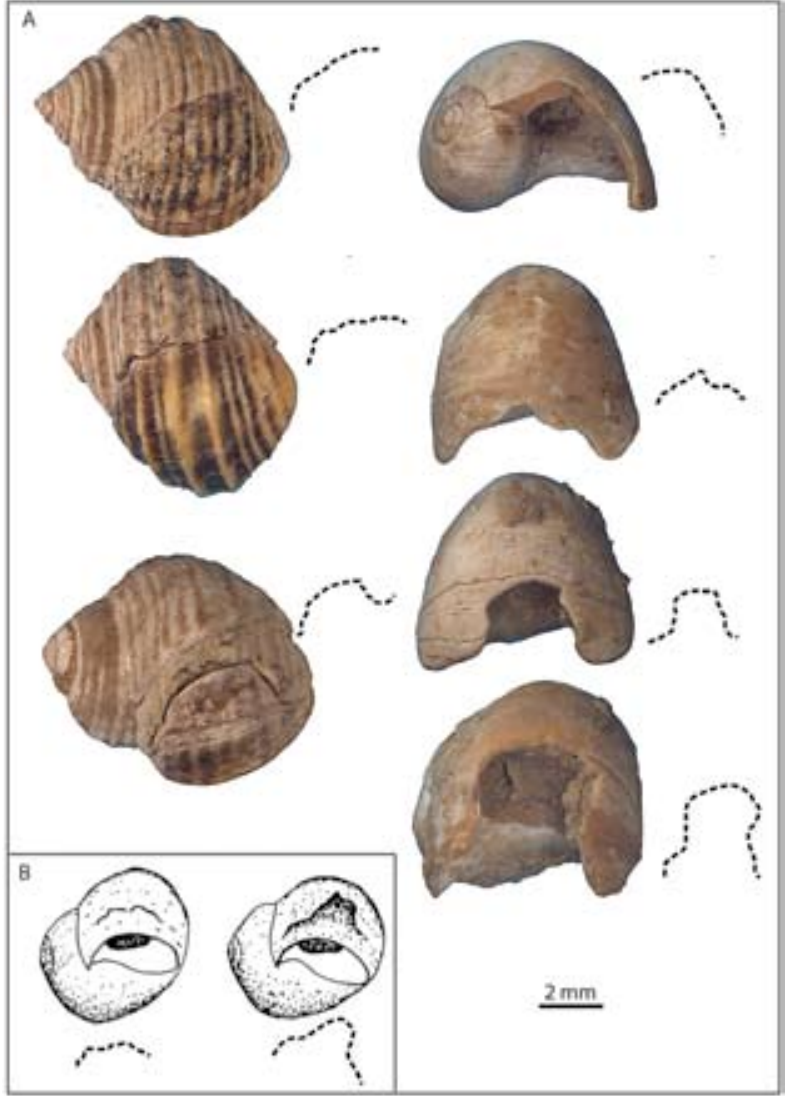

Fig. 13. A) Comparaison des traces de fractures présentes sur les coquilles de mollusques vivants et des coquilles de Littorines provenant de Praileaitz : à gauche, cassures résorbées observées sur les coquilles du référentiel de $L$. saxatilis collectées vivantes sur la plage de Paloma ; à droite : cassures non résorbées observées sur les coquilles de Praileaitz. B) Morphologie des cassures résorbées résultants de l'action du ressac ou de la prédation observées sur des Littorines des îles britanniques (d'après REIMCHEN 1982). / Comparison of the shape of reabsorbed fractures observed on modern shells and the breakage recorded on the Littorina from Praileaitz: left, reabsorbed fractures on modern $L$. saxatilis C. collected at Paloma, right: breakage recorded on shells from Praileaitz. B) Morphology of reabsorbed fractures resulting from surf or predation on modern Littorina from the British Isles (REIMCHEN, 1982).

indiquent qu'elles ne partagent pas entièrement le même biotope. Aussi, la collecte de ces deux espèces vivantes nécessite soit l'exploitation d'une plage présentant simultanément les caractéristiques topographiques et environnementales nécessaires au développement des deux espèces, soit la pratique d'une collecte des coquillages à des endroits différents correspondant au biotope de chacune des deux espèces. En revanche, les thanatocénoses peuvent regrouper sur des plages abritées des espèces se développant dans des environnements très variés de leur vivant.

Des coquillages échoués et accumulés au sein des thanatocénoses présentent des caractéristiques particulières à leur surface. Notamment, le ressac entraîne la modification de la forme générale, de l'ornementation et des reliefs des tests (TABORIN, 1993, LOZOUET, VIGNE, 1994). Pour les gastéropodes, les parties convexes telles que la spire la plus large et l'apex, seront usées par le frottement sur le sable (DUPONT, 2006). Aucune de ces caractéristiques n'a été observée sur les coquilles de Praileaitz I. En revanche, les cassures présentes sur plus de la moitié des pièces, qui consistent en la disparition d'une écaille plus ou moins envahissante sur le bord dorsal de l'aperture des coquilles, sont caractéristiques des marques de la prédation des gastéropodes par des crabes (REIMCHEN, 1982, CROTHERS, 2003). Lorsque l'attaque échoue, le test du coquillage peut se réparer (BLUNDON, VERMEIJ, 1983, CROTHERS, 2003) et une délimitation marquant l'ancienne cassure du coquillage dans les stries de croissances des coquilles est visible (Fig. 13). Ce type de fracture peut aussi résulter de l'action d'un intense ressac (REIMCHEN, 1982).

L'absence de trace de résorption sur les coquilles fracturées de Praileaitz I plaide pour une attaque de crabe réussie, ayant conduit à la mort des animaux. De même, les petites perforations coniques observées sur la face ventrale de deux coquillages sont caractéristiques de la prédation du coquillage par un organisme perforateur (D'ERRICO et al., 1993, TABORIN, 1993, DUPONT, 2006). Ces éléments suggèrent que les coquillages ont été collectés au sein de leur thanatocénose, accumulée sur une plage relativement abritée. Dans ce contexte, l'absence des traces caractéristiques de l'usure mécanique des coquilles soumises au ressac lorsque celle-ci sont accumulées au sein des thanatocénoses, suggère que seules les coquilles présentant des surfaces fraîches, probablement en raison d'une mort du mollusque peu de temps avant le dépôt au sein de la thanatocénose, ont été collectées et importées sur le gisement de Praileaitz l.

\subsection{Selection}

La collecte d'individus morts exclut l'hypothèse alimentaire pour expliquer la présence des coquillages dans la cavité. Dans ce contexte, si l'hypothèse d'une fonction utilitaire peut être abandonnée, l'hypothèse d'une fonction symbolique, telle qu'une utilisation en tant qu'ornement corporel doit être explorée.

L'analyse morphométrique montre que les L. obtusata accumulées à Praileaitz I sont significativement plus petites que les coquillages de la même espèce constituant les référentiels de comparaison actuels. Les $L$. obtusata de Praileaitz I sont également significativement plus petites que les coquillages des référentiels archéologiques attribués au Magdalénien supérieur et inférieur. Les $L$. saxatilis, quant à elles, couvrent la variabilité métrique du référentiel actuel de comparaison mais présentent une proportion de pièces significativement plus grandes que les pièces du référentiel actuel. Les deux $L$. saxatilis provenant des gisements attribués au Magdalénien, ou à l'Azilien pour la pièce des déblais de Urtiaga, s'intègrent à la variabilité métrique des coquilles de la même espèce provenant de Praileaitz I et du référentiel actuel. 
Différents paramètres environnementaux peuvent être à l'origine de variations métriques et morphologiques des coquilles de littorines. Des coquillages d'une même espèce peuvent se distinguer en différents écotypes, selon la température de l'eau dans laquelle ils se développent, l'intensité des variations saisonnières, l'intensité du ressac et l'intensité de la prédation (NEWKIRK, DOYLE, 1975, JOHANNESSON et al., 1993, REID, 1993, QUEIROGA et al., 2011). Dans ce contexte, deux facteurs peuvent être à l'origine des différences de tailles observées entre la collection archéologique de Praileaitz I et les différents ensembles archéologiques et actuels de comparaison: une sélection anthropique des pièces en fonction de la taille des coquilles, ou une évolution dans le temps de la variabilité métrique des pièces de chacun des ensembles qui, en raison de leur attribution chronologique différente et de leur origine géographique différente, n'ont pas été soumises aux mêmes contraintes environnementales de leur vivant.

De manière générale, au cours du Pléistocène supérieur, la taille des coquillages a tendance à diminuer avec l'augmentation de la température des eaux de surface (BAILEY, CRAIGHEAD, 2003, GUTIERREZ-ZUGASTI, 2011). Visible au cours des phases interstadiaires, ce phénomène semble s'accentuer à partir du début de l'Holocène par l'intensification de l'exploitation des ressources malacologiques, combinée à l'augmentation de la température (GUTIERREZ-ZUGASTI, 2011). Des différences dans la taille des coquillages peuvent aussi résulter des variations de la température de l'eau et de variations saisonnières (NEWKIRK, DOYLE, 1975, REID, 1993, QUEIROGA et al., 2011). Les grandes dimensions enregistrées sur les $L$. obtusata provenant des couches magdaléniennes et épipaléolithiques des sites basques étudiés semble être en cohérence avec l'hypothèse climatique. En revanche, le fait que les Littorina obtusata de Praileaitz I soient significativement plus petites que les $L$. obtusata actuelles est en contradiction avec cette hypothèse. Cela semble indiquer que seules les plus petites L. obtusata présentes dans l'environnement de Praileaitz I au moment de l'accumulation sont représentées sur le gisement. Une telle distribution de taille pourrait être le témoin d'un tri anthropique des pièces selon leur dimension. Même si elle nous semble la plus probable, cette hypothèse doit cependant être nuancée. Les référentiels de comparaison archéologiques utilisés proviennent tous les deux de la région vasco-cantabrique. En revanche, les référentiels actuels de comparaison proviennent de latitudes plus nordiques. Ce décalage latitudinal pourrait être la raison de la différence de taille observée entre les populations actuelles et l'échantillon archéologique. II est donc nécessaire, avant de conclure à une préférence pour une taille particulière de $L$. obtusata d'élargir le référentiel.

Les L. saxatilis de Praileaitz I, quant à elles, sont significativement plus grandes que celles composant le référentiel actuel collecté en Asturie. Cette différence est tout à fait en accord avec l'hypothèse d'une réduction de la taille des coquillages corrélée à l'augmentation de température holocène (GUTIERREZ-ZUGASTI, 2011). Si tel est le cas, les coquillages appartenant au complexe des $L$. saxatilis présents à Praileaitz I n'ont pas fait l'objet d'un tri orienté vers les plus petits coquillages.

Pour résumer, les données métriques suggèrent que seules les L. obtusata pourraient avoir fait l'objet d'une sélection selon leur taille à Praileaitz I, bien que l'hypothèse environnementale pour expliquer les différences avec les référentiels actuels ne puisse être exclue pour l'instant. En revanche, les $L$. saxatilis ne semblent pas avoir fait l'objet d'une telle sélection.

\subsection{Transformation}

Aucune transformation vouée à aménager un système de suspension sur les coquilles n'a été observée. Cependant, la sélection des pièces présentant des surfaces bien conservées au sein de leur thanatocénose, leur probable tri selon leur dimension et la présence quasi exclusive de ces deux espèces sur le gisement semblent bien la preuve que cette accumulation est le résultat d'une action anthropique délibérée.

\subsection{Attribution chronologique}

Aucun artefact diagnostique d'un faciès culturel particulier n'a été collecté dans le niveau d'occupation de Praileaitz I ayant livré les coquillages. De ce fait, l'attribution chronologique de cet ensemble doit se baser sur l'attribution chrono-culturelle des couches sous- et sus-jacentes, qui ont livré du matériel archéologique, et s'appuyer sur les datations ${ }^{14} \mathrm{C}$ obtenues pour ces couches ainsi que sur les âges ${ }^{14} \mathrm{C}$ obtenu sur les coquillages composant cet assemblage.

À l'exception de six pièces présentes dans les bandes $\mathrm{G}, \mathrm{C}, \mathrm{H}$ et $\mathrm{F}$, qui apparaissent clairement isolées du reste de l'accumulation, qui s'étend principalement sur les bandes $A^{\prime}$ à $H^{\prime}$, la dispersion des coquilles est restreinte à la zone située entre la première et la deuxième salle. L'accumulation des coquilles présente ainsi une certaine homogénéité spatiale, qui combinée à l'absence de vestige de cette nature dans les niveaux archéologiques encadrant ce dépôt, suggère qu'il n'y a pas d'incohérence stratigraphique majeure. Ces données spatiales cohérentes font fortement écho à la grande homogénéité du matériel malacologique lui-même, composé presque exclusivement de deux seules espèces. Le niveau ayant livré les coquillages est surmonté d'un niveau riche en charbons daté entre 10217 et 9734 cal BP (Tabl. 4, PEÑALVER IRIBARREN, MUJIKA ALUSTIZA, 2003, PEÑALVER IRIBARREN, SAN JOSE SANTAMARTA, 2008). Ces âges, parfaitement cohérents, fixent un clair terminus ante quem à l'accumulation de coquillages. Les âges compris entre 19397 et 18578 cal BP livrés par la couche sous-jacente, attribuée au Magdalénien inférieur 
(PEÑALVER IRIBARREN, MUJIKA ALUSTIZA, 2003, PEÑALVER IRIBARREN, SAN JOSE SANTAMARTA, 2008) fixent de leur côté un clair terminus post quem. Établir avec certitude, au sein de cet intervalle, l'âge de l'accumulation est plus problématique. Les âges obtenus directement sur les coquillages ne se recoupent pas et couvrent à première vue une plage de presque 2000 ans. La collecte des coquilles au sein de thanatocénoses pourrait expliquer cet écart. Les accumulations naturelles de coquillages peuvent être préservées pendant de longues périodes de temps, atteignant dans certains cas, plusieurs milliers d'années. La collecte d'un ensemble de coquillages peut ainsi être largement postérieure à la formation de la thanatocénose. L'écart entre les dates obtenues sur les deux coquilles de Praileaitz pourrait refléter leur collecte au sein de deux thanatocénoses distinctes et non contemporaines. L'écart entre les dates pourrait aussi être interprété comme la preuve d'une fréquentation prolongée du site pour y mener une activité ciblée. Cependant, l'absence d'autres vestiges archéologiques dans cette couche, la cohérence observée dans la distribution verticale des coquillages, le fait que la date la plus récente se superpose partiellement à celles obtenues pour la couche sus-jacente, et les problèmes bien connus liés à la datation ${ }^{14} \mathrm{C}$ des coquillages (DOUKA et al., 2010a, b), abondent pour une chronologie courte et un âge ancien pour l'accumulation des coquillages. Si cette hypothèse est confirmée par de nouvelles datations, les littorines auraient été abandonnées à Praileaitz I à la fin du Dryas récent (12,8-11,5 ka cal $\mathrm{BP}$ ) ou à la charnière Dryas récent-Holocène. En considérant les âges obtenus dans les sites de la côte vasco-cantabrique pour les technocomplexes de cette période (GONZALEZ MORALES et al., 2004), les auteurs de cette accumulation seraient probablement des chasseurs Aziliens.

\begin{tabular}{|l|l|l|l|l|}
\hline Lab code & Couche & Âge conventionnel & cal BP Corr & Attribution \\
\hline GrA-28028: & Charbonneuse & $8940 \pm 50 \mathrm{BP}$ & $10217-9912$ & Epipaléolithique \\
\hline GrA-28030 & Charbonneuse & $8840 \pm 45 \mathrm{BP}$ & $10157-9734$ & Epipaléolithique \\
\hline GrA-38266: & Charbonneuse & $8845 \pm 40 \mathrm{BP}$ & $10157-9741$ & Epipaléolithique \\
\hline KIA-39657 & Coquillages & $9320 \pm 40 \mathrm{BP}$ & $10571-10252$ & Epipaléolithique \\
\hline KIA-39658 & Coquillages & $10560 \pm 50 \mathrm{BP}$ & $12621-12114$ & Epipaléolithique \\
\hline GrA-24685 & Jaune & $15530 \pm 100 \mathrm{BP}$ & $18982-18578$ & Magdalénien inférieur \\
\hline GrA-24688 & Jaune & $15810 \pm 110 \mathrm{BP}$ & $19397-18827$ & Magdalénien inférieur \\
\hline
\end{tabular}

Tabl. 4. Âges radiocarbone obtenus sur les deux coquillages marins de Praileaitz I et les couches sus- et sous-jacentes. / Radiocarbon ages obtained on shells from Praileaitz I and for the overlying and underlying stratigraphic units.

\section{5.- CONCLUSION}

Les Littorina saxatilis et des Littorina obtusata sont utilisées de façon presque ininterrompue au sein des parures des sociétés ayant occupé la région vasco-cantabrique depuis le début du Paléolithique supérieur jusqu'au Mésolithique. Cependant, les études ayant contribué à la caractérisation des stratégies d'approvisionnement et de gestion de ces matériaux, permettant ainsi des inférences quant à l'organisation sociale et symbolique des groupes au sein de leur territoire sont rares. En croisant des données éthologiques, morphométriques et taphonomiques nous mettons en évidence des activités spécialisées dans la confection d'ornements personnels et suggérons l'existence de sites spécialisés ayant pu jouer un rôle spécifique dans l'économie et l'organisation des sociétés épipaléolithiques du Nord de la façade atlantique de la péninsule ibérique.

Notre analyse propose que ces deux espèces ont été exclusivement sélectionnées au sein de leur thanatocénose et que, au cours de la collecte, une attention particulière a été donnée aux coquillages présentant un état de surface relativement frais. De plus, seules les $L$. obtusata les plus petites sont présentes sur le gisement et presque la moitié des coquilles des deux espèces montrent une cassure au bord de leur aperture.

Partant du constat que l'utilisation de ces espèces pour en faire des objets de parure est documentée à l'Epipaléolithiques et que l'on ne leur connaît aucune autre fonction, l'accumulation de Praileaitz I semble être constituée de pièces qui ont été collectées dans l'objectif de les intégrer à une parure, mais abandonnées sur le gisement sans modification.

Deux hypothèses peuvent être proposées pour expliquer l'abandon des pièces sans aménagement :

- les pièces de Praileaitz I constituent une réserve de matière première, vouée à être transformées plus tard en objet de parure,

- ces pièces ont été considérées par les occupants du site comme inappropriées pour être intégrées à une parure et ont été abandonnées en l'état sur le gisement. 
La différence de taille que l'on observe entre les coquillages de Paraileaitz I et les objets de parure confectionnés avec les mêmes coquillages dans des sites proches géographiquement et chronologiquement plaide pour la seconde hypothèse. L'accumulation des L. obtusata semble résulter d'un tri des pièces selon leur dimension avec l'objectif d'exclure des coquillages de trop petites dimensions.

Les résultats obtenus suggèrent ainsi qu'une partie des activités menées par les chasseurs-cueilleurs qui ont occupé le gisement de Praileaitz I était vouée à la confection d'ornements. La présence de rejets de matière première et l'exportation de coquillages dédiés à être transformés en objets de parure par la suite, montrent une segmentation dans l'espace de la chaîne opératoire de confection de ces ornements corporels. L'absence de tout autre vestige archéologique associé avec les coquillages plaide pour des activités de tris réalisées au cours d'haltes brèves par un groupe dont le principal ou l'unique objectif était l'acquisition de supports voués à la production d'objets symboliques. La nature très spécialisée de cette activité est à mettre en exergue. Si des ornements réalisés sur les mêmes supports sont attestés dans d'autres gisements, contemporains et plus anciens, de la région (ÁLVAREZ-FERNANDEZ, 2006), Praileaiz est le seul site qui permet à la fois de déterminer les modalités et lieux de collecte, de documenter en détail une étape de production des parure en coquillage, d'identifier les critères de sélection et l'insérer dans cette étape de production dans le parcours des chasseurs-cueilleurs de cette époque.

\section{6.- REMERCIEMENTS}

Nous remercions Xabier Peñalver et Sonia San Jose pour nous avoir confié l'étude de la collection de coquillages marins de Praileaitz I, pour leur aide et pour leur accueil chaleureux à San Sebastián. Nous remercions également E. Álvarez-Fernández et I. Gutiérrez pour avoir attiré notre attention sur certaines références bibliographiques et pour les échanges enrichissants que nous avons eus au cours de l'étude de ces coquillages. Nous sommes également reconnaissants envers Koro Mariezkurrena-Gaztearena pour son accueil au dépôt de fouille de Patrimonio del Gobierno Vasco à San Sebastián et pour nous avoir permis l'accès aux collections de Urtiaga et Erralla. Cette recherche a été financée par le programme européen Origin of Man, Language and Languages

\section{7.- BIBLIOGRAPHIE}

ALTUNA, J., BALDEÓN, A., MARIEZKURRENA, K.

1985 Cazadores magdalenienses en la cueva de Erralla (Cestona, Pais Vasco). Munibe Antropologia-Arkeologia 37, 206.

ALTUNA-ETXABE, J., MARIEZKUURRENA-GAZTEARENA, K.

2010 Moluscos marinos de la Cueva de Urtiaga. Kobie Serie Paleoantropología 29, 47-64.

\section{ÁLVAREZ-FERNÁNDEZ E.}

2001 L'axe Rhin-Rhône au Paléolithique supérieur récent l'exemple des mollusques utilisés comme objets de parure. L'Anthropologie 105, 547-64.

2006 Los objetos de adorno-colgantes del Paleolitico superior y del Mesolitico en la Cornisa Cantabrica y en el Valle del Ebro: una vision europea. Universidad de Salamanca. Salamanca.

2007 The exploitation of marine molluscs in Cantabrian Spain during the Gravettian: first results of Layers E \& F of La Garma A (Omoño, Cantabria). Zephyrus 60, 43-58.

2009 Análisis arqueomalacológico de la cueva de Altamira (Santillana del Mar, Cantabria): Excavaciones de J. González Echegaray y L.G. Freeman. Complutum 20, 55-70.

2010 Limpets \& Periwinkles in Cantabrian Spain between 22,000 and 15,000 Cal BC: Archaeomalacological Remains at Altamira Cave. Revista Facultății de Istorie a Universităţii Creştine Dimitrie Cantemir, Serie Istorie1 Serie Noua 1, 32-51.

2011a Humans and marine resource interaction reappraised: Archaeofauna remains during the late Pleistocene and Holocene in Cantabrian Spain. Journal of Anthropologica Archaeology 30, 327-43.

2011b Mollusc shell sizes in archaeological contexts in Northern Spain (13 200 to 2600 cal BC): New data from la Garma A and los Gitanos (Cantabria). Archaeometry 53, 963-985.

BAILEY G.N., CRAIGHEAD, A.S,

2003 Late Pleistocene and Holocene coastal palaeoeconomies: A reconsideration of the molluscan evidence from northern Spain. Geoarchaeology 18, 175-204

BLUNDON, J.A., VERMEIJ, G.J.

1983 Effect of shell repair on shell strength in the gastropod Littorina irrorata. Marine Biology 76, 41-5.

CAZALS, N.

2005 Le début du Magdalénien de part et d'autre des Pyrénées. Quelques réflexions au travers des techniques de taille et des modes d'exploitation des ressources, en JAUBERT, J., BARBAZA, M. (Eds). Territoires, déplacements, mobilité, échanges durant la Préhistoire, 126e Congrès du CTHS, Toulouse 2001. 295-309. CTHS. Paris.

CONDE-PADIIIN, P., GRAHAME, J.W., ROLÁN-ALVAREZ, E.

2007 Detecting shape differences in species of the Littorina saxatilis complex by morphometric analysis. Journal of Molluscan Studies 73, 147-54.

CORCHÓN, S., MATEOS, A., ÁLVAREZ FERNÁNDEZ, E., PEÑALVER, E., DELCLÒS, X., VAN DER MADE, J.

2008 Ressources complémentaires et mobilité dans le Magdalénien cantabrique. Nouvelles données sur les mammifères marins, les crustacés, les mollusques et les roches organogènes de la Grotte de Las Caldas (Asturies, Espagne). L'anthropologie 112, 284-32. 
CROTHERS, JH.

2003 Rocky shore snail as material for projects (With a key for their identification). Field Studies 10, 601-34.

D'ERRICO, F., JARDON-GINER, P., SOLER-MAYOR, B.

1993 Critères à base expérimentale pour l'étude des perforations naturelles et intentionnelles sur coquillages, en Traces et fonction: les gestes retrouvés. 244-53. CTHS. Liège.

D'ERRICO, F., VANHAEREN, M., QUEFFELEC, A.

2017 Les galets perforés de Praileaitz I (Deba, Gipuzkoa), en PEÑALVER, X., SAN JOSE, S., MUJIIKA-ALUSTIZA, J.A. La cueva de Praileaitz I (Deba, Gipuzkoa, Euskal Herria). Intervención arqueológica 2000 - 2009. Munibe Monographs. Anthropology and Archaeology Series 1, 453484.

DOUKA, K., HEDGES, R.E.M., HIGHAM, T.F.G.

2010 Improved AMS 14C dating of shell carbonates using high-precision $\mathrm{X}$-ray diffraction $(\mathrm{XRD})$ and a novel density separation protocol (CarDS). Radiocarbon 52, 735-51.

DOUKA, K., HIGHAM, T.F.G., HEDGES, R.E.M.

2010 Radiocarbon dating of shell carbonates: old problems and new solutions. Munibe. Suplemento 31, 18-27.

DUPONT, C.

2006 La malacofaune de sites mésolithiques et néolithiques de la façade atlantique de la France: Contribution à l'économie et à l'identité culturelle des groupes concernés. Oxford. (British Archaeological Reports, Archeopress, International Series; 1571).

FULLOLA, J.M., MANGADO, X., ESTRADA, A.

2007 Circulation des matières premières lithiques et des coquillages dans le Paléolithique supérieur du Nord-Est ibérique, en La notion de mobilité dans les sociétés préhistoriques. 57-66. CTHS. Paris.

GAILLARD, JM.

1977 Le polymorphisme de Littorina saxatilis. Malacologia 16, $11-4$.

GALPARSORO, I., BORJA Á., LEGORBURU I., HERNANDEZ C., CHUST G. et al.

2010 Morphological characteristics of the Basque continental shelf (Bay of Biscay, northern Spain) ; their implications for Integrated Coastal Zone Management. Geomorphology $118,314-29$.

GONZÁLEZ MORALES, M.R., STRAUS, L. G., DÍEZ CASTILLO, A., RUIZ COBO, J.

2004 Postglacial Coast and Inland: The Epipaleolithic-Mesolithic-Neolithic Transitions in the Vasco-Cantabrian Region. Munibe Antropologia-Arkeologia 56, 61-78.

GUTIÉRREZ-ZUGASTI, I.

2009a An examination of Mesolithic shellfishing activities in the lower Asón river basin (Cantabria, Spain), in MCCARTAN, S., SCHULTING, R.J., WARREN, G., WOODMAN, P. Mesolithic Horizons. Proceedings of the VII International Conference on the Mesolithic in Europe. 161-166. Oxbow Books. Belfast. 2009b La explotación de moluscos y otros recursos litorales en la región cantábrica durante el Pleistoceno final y el Holoceno inicial. Universidad de Cantabria. Santander.

2011 Coastal resource intensification across the PleistoceneHolocene transition in Northern Spain: Evidence from shell size and age distributions of marine gastropods. Quaternary International 244, 54-66.

HARASEWYCH, J., MOREIZSOHN, F.

2010 The Book of Shells: A Life-Size Guide to Identifying and Classifying Six Hundred Seashells. University of Chicago Press.

JOHANNESSON, K., JOHANNESSON, B., ROLÀN-ALVAREZ, E.

1993 Morphological differentiation and genetic cohesiveness over a microenvironmental gradient in the marine snail Littorina Saxatilis. Evolution 47, 1770-87.

JOHANNESSON, K., EKENDAHL, A.

2002 Selective predation favoring cryptic individuals of marine snails (Littorina). Biological Journal of the Linnean Society $76,137-44$.

LAMBECK, K., CHAPPEL, J.

2001 Sea level change through the last glacial cycle. Science 292.

LAMBECK, K., ESAT, T.M., POTTER, E.-K.

2002 Links between climate and sea levels for the past three million years. Nature 419,199-205.

LANGLAIS, M.

2010 Les sociétés magdaléniennes de l'isthme pyrénéen. CTHS. Paris.

LEORRI, E., CEARRETA, A., MILNE G.

2012 Field observations and modelling of Holocene sea-level changes in the southern Bay of Biscay: implication for understanding current rates of relative sea-level change and vertical land motion along the Atlantic coast of SW Europe. Quaternary Science Reviews 42, 59-73.

LOZOUET, P., VIGNE, J-D.

1994 Les invertébrés, in VIGNE, J.D. (Ed.). Lîle de Lavezzi, Hommes, animaux, archéologie et marginalité (XIII, XX s., Bonifacio, Corse). 155-64. Éditions CNRS. Paris.

MONTEIRO CARVALHO, JG.

2014 Study on the diversification of flat periwinkles (Littorina fabalis and L. obtusata): insights from genetics and geometric morphometrics. Mestrado em Biologia Evolutiva e do Desenvolvimento. Lisboa.

NEWIKIRK, G., DOYLE, R.

1975 Genetic analysis of shell shape variation in Littorina saxatilis on an environmental cline. Marine Biology 30, 227-37. 
NIIES, L.H.P., SITTERS, A., DEY, A., BAKER, R.I.G., MORRISON, D. et al.

2006 Status of the Red Knot (Calidris canutus rufa) in the Western Hemisphere. New Jersey Department of Environmental Protection, Division of Fish and Wildlife, Endangered and Nongame Species Program, Trenton. New Jersey.

PANOVA, M., BLAKESLEE, A., MILLER, A., MÄKINEN, T., RUIZ, G. et al.

2011 Glacial History of the North Atlantic Marine Snail, Littorina saxatilis, Inferred from Distribution of Mitochondria DNA Lineages. PLoSONE 6. doi:10.1371/journal.pone.0017511.

PEÑALVER IRIBARREN, X., MUJIKA ALUSTIZA, J.A.

2003 Suelo de ocupación magdaleniense en la cueva de Praile Aitz I (Deba, Gipuzkoa): evidencias de arte mobiliar. Veleia 20, 157-81.

PEÑALVER IRIBARREN, X., SAN JOSE SANTAMARTA, S.

2008 La grotte de Praileaitz I (Deba, Gipuzkoa, Euskal Herria Pays Basque et la défense du patrimoine dans le Pays Basque). Préhistoire du Sud-Ouest 16, 143-50.

POPPE, T., GOTO, Y.

1991 European Seashells. Vol. I: Polyplacophora, Caudofoveata, Solenogastra, Gasteropoda. Verlag Christa. Hemmen.

POPPE, G.T., GOTO, Y.

1993 European Seashells. Vol. II : Scaphopoda, Bivalvia, Cephalopoda. Verlag Christa. Hemmen.

QUEIROGA, H., COSTA, R., LEONARDO, N., SOARES, D., CLEARY, D.F.R.

2011 Morphometric variation in two intertidal littorinid gastropods. Contributions to Zoology 80, 201-11.

REID, D.G.

1993 Barnacle-dwelling ecotypes of three British Littorina species and the status of Littorina neglecta Bean. Journal of Molluscan Studies 59, 51-62.

1996 Systematic and evolution of Littorina. The Ray Society. London.

REIMCHEN, T.E.

1982 Shell size divergence in Littorina mariae and L. obtusata and predation by crabs. Canadian Journal of Zoology 60, 687-695.

RIGAUD, S., GUTIÉRREZ-ZUGASTI, I.

2016 Symbolism among the last hunter-fisher-gatherers in northern Iberia: Personal ornaments from El Mazo and El Toral III Mesolithic shell midden sites. Quaternary International 407, Part B, 131-144. doi:10.1016/j. quaint.2015.10.029.

RIGAUD, S., D'ERRICO, F., VANHAEREN, M., PEÑALBER, X.

2014 A short-term, task-specific site: Epipalaeolithic settlement patterns inferred from marine shells found at Praileaitz I (Basque Country, Spain). Journal of Archaeological Science 41, 666-678.
STRAUS, L.G.

1991 Epipaleolithic and Mesolithic Adaptations in Cantabrian Spain and Pyrenean France Journal of World Prehistory 5, 83-104.

2005 The Upper Paleolithic of Cantabrian Spain. Evolutionary Anthropology 14, 145-58.

STRAUS, L.G., GONZALEZ MORALES, M.R., FANO MARTINEZ, M.A., GARCIA-GELABERT, M.P.

2002 Last Glacial Human Settlement in Eastern Cantabria (Northern Spain). Journal of Archaeological Science 29, 1403-14

STUIVER, M., REIMER, P.J.

1993 Extended 14c data base and revised calib 3.014c age calibration program. Radiocarbon 35, 215-230.

TABORIN, Y.

1993 Traces de façonnage et d'usage sur les coquillages perforés, en ANDERSON, P., BEYRIES, S., OTTE, M., PLIS$\mathrm{SON}, \mathrm{H}$. (eds). Traces et fonction : les gestes retrouvés. 255-67. ERAUL. Liège.

1996 La parure paléolithique et la notion de territoire, en XIII International Congress of Prehistoric and Protohistoric Sciences, 8-145 sept. 1996, colloquium XVI. 143-8. Forli, Italie.

TREWIN, N.H., WELSH, W.

1976 Formation and composition of a graded estuarine shell bed. Palaeogeography, Palaeoclimatology, Palaeoecology 19, 219-30

UTRILLA, P.

1996 La sistematización del Magdaleniense cantábrico: una revisión histórica de los datos, en MOURRE ROMANILLO A. (ed.). El hombre fósil 80 años después, Homenaje a Hugo Obermaier. 211-47. Universidad de Cantabria.

VANHAEREN, M.

2002 Les fonctions de la parure au Paléolithique supérieur : de l'individu à l'unité culturelle. Thèse d'Université. Université de Bordeaux 1. Talence.

2010 Les fonctions de la parure au Paléolithique supérieur : de l'individu à l'unité culturelle. Editions Universitaires Européennes. Sarrebruck.

VANHAEREN, M., D'ERRICO, F.

2003 The Body Ornaments Associated with the Burial, en ZILHAO, J., TRINKAUS, E. (eds.). Portrait of the Artist as a Child. The Gravettian human skeleton from the Abrigo do Lagar Velho and its archaeological context. 154-86. Instituto Portugues de Archeologia. Lisbonne.

2005 Grave goods from the Saint-Germain-la-Rivière burial: Evidence for social inequality in the Upper Palaeolithic. Journal of Anthropological Archaeology 24, 117-34. 
